\title{
COMPLEX ANALYTIC DYNAMICS ON THE RIEMANN SPHERE
}

BY PAUL BLANCHARD

\section{Table of Contents}

1. Background and notation

2. The dynamical dichotomy of Fatou and Julia

3. Periodic points

4. The consequences of Montel's Theorem

5. The Julia set is the closure of the set of repelling periodic points

6. Classical results concerning the Fatou set

7. Sullivan's classification of the Fatou set

8. A condition for expansion on the Julia set

9. The dynamics of polynomials

10. The Mandelbrot set and the work of Douady and Hubbard

11. The measurable Riemann mapping theorem and analytic dynamics

12. Bibliographic notes

List of notation

References

Holomorphic, noninvertible dynamical systems of the Riemann sphere are surprisingly intricate and beautiful. Often the indecomposable, completely invariant sets are fractals (à la Mandelbrot [M1]) because, in fact, they are quasi-self-similar (see Sullivan [S3] and (8.5)). Sometimes they are nowhere differentiable Jordan curves whose Hausdorff dimension is greater than one (Sullivan [S4] and Ruelle [R]). Yet these sets are determined by a single analytic function $z_{n+1}=R\left(z_{n}\right)$ of a single complex variable.

The study of this subject began during the First World War. Both P. Fatou and G. Julia independently published a number of Compte Rendu notes, and then both wrote long memoires - Julia [J] in 1918 and Fatou [F1-F3] in 1919 and 1920. At that time, they had at their disposal a new theorem of Montel (see (4.1)) which gave a sufficient condition for the normality of a family of meromorphic functions. They applied the theory of normal families to the dynamical system to prove some remarkable results.

Received by the editors December 7, 1983.

1980 Mathematics Subject Classification. Primary 58Fxx; Secondary 30D05. 
Recently there has been an explosion of interest in the subject, and many mathematicians have made substantial contributions. In fact, significant progress is still being made, and it is impossible to predict where and when it will end. The aim here is to give a rapid introduction to the subject in light of these achievements. We do not intend to survey most of the new work since much of it is still in progress, but we do include bibliographic notes (\$12) which can serve as a reader's guide to those results with which we are familiar. However, no claims of completeness are implied or intended.

A large part of this paper is an exposition of the classical work of Fatou and Julia. We give a complete proof of the fundamental result (Theorem (5.15)) that the Riemann sphere disjointly splits into two sets-the closure of the repelling periodic points and the open set of normal (i.e. stable) points. Along the way, we discuss the dynamics in a neighborhood of a periodic point ( $\$ 3)$ and the global consequences of Montel's Theorem (\$4). The decomposition theorem is proven in $\$ 5$. $\$ \S 6$ and 8 contain other noteworthy classical results. The theorems in $\$ 9$ were also known classically, although they were not formulated in quite the same manner.

$\S \S 7,10$ and 11 contain expositions of recent work. Sullivan [S1, S2] has completed a classification of the dynamics in the domains of normality. We summarize this classification in $\$ 7$ and briefly indicate how the theory of quasi-conformal homeomorphisms plays a crucial role in $\$ 11 . \S 10$ is an exposition of the recent work of Douady and Hubbard [DH] and Mandelbrot [M1, M2] on the dynamics of quadratic polynomials. A brief survey of this work has been included because it is a wonderful example of how "simple" dynamical systems can have complicated dynamics. Moreover, the family of quadratic polynomials is varied enough to illustrate most of the ideas presented here as well as many other surprising phenomena. Although the material in $\S \S 7,9$ and 10 depends on the previous sections, the reader may find it helpful to read these sections before tackling some of the more involved proofs in the preceding ones.

The first section simply establishes notation and recalls theorems from complex analysis and the theory of Riemann surfaces which will be used freely throughout the article. A list of notation has been provided for readers who would like to skip this section.

Acknowledgements. This paper was written while the author was a postdoctoral fellow, first at the Institute for Mathematics and its Applications, then at the Institute for Advanced Study. The author would like to thank the many people at both institutions who created such pleasant environments in which to work. Both fellowships were supported by the National Science Foundation.

The Dynamical Systems Laboratory at the University of Minnesota provided the author with unlimited access to valuable graphics equipment. Without this equipment, it would have been impossible to view any but the most elementary Julia sets. The final pictures were made in the Dynamical Systems Laboratory at Boston University. The equipment at Boston University was provided through grants from the National Science Foundation and Boston University. 
This paper is based on notes from two seminars-one at Boston University and the other at the Institute for Mathematics and its Applications. Both seminars owed a great deal to Brolin's expository article [B] as well as to the participants. The author particularly wants to acknowledge many informative conversations with P. Collet, R. Crew, R. Devaney, M. Handel, O. Lanford, R. McGehee, R. Moeckel, B. Osgood, R. Penner, M. Rees, C. Tresser, and R. Williams, and he would like to thank A. Douady and J. Hubbard for spending a good deal of time explaining their results to him.

The author would like to especially thank Dennis Sullivan, whose lectures introduced him to this beautiful subject.

1. Background and notation. We are interested in studying the dynamics of a discrete dynamical system of the Riemann sphere $\overline{\mathbf{C}}$ generated by a holomorphic transformation

$$
R: \overline{\mathbf{C}} \rightarrow \overline{\mathbf{C}} \text {. }
$$

In other words, our phase space will be the unique, simply connected, closed Riemann surface $\overline{\mathbf{C}}=\mathbf{C} \cup\{\infty\}$ which is homeomorphic to the two-dimensional sphere

$$
S^{2}=\left\{\left(x_{1}, x_{2}, x_{3}\right) \in \mathbf{R}^{3} \mid x_{1}^{2}+x_{2}^{2}+x_{3}^{2}=1\right\} .
$$

We shall usually use the variables $z$ and $w=1 / z$ to represent the two standard coordinate charts on $\overline{\mathbf{C}}$ determined by stereographic projection. Then any holomorphic (analytic) map $R$ of $\overline{\mathbf{C}}$ can be written in the form

$$
R(z)=p(z) / q(z)
$$

where $p(z)$ and $q(z)$ are polynomials with complex coefficients and no common factors. Hence, there is a one-to-one correspondence between rational functions (1.2) and holomorphic maps (1.1). The poles of the rational function are simply the points of $\overline{\mathbf{C}}$ which are mapped to infinity. The reader should consult Hille [Hi], Ahlfors [A1], Narasimhan [N], or Farkas and Kra [FK] for more details.

The degree, $\operatorname{deg}(R)$, of any continuous map $R: S^{2} \rightarrow S^{2}$ is a homotopy invariant which measures how many times $R$ wraps $S^{2}$ around itself. In our context the degree of $R$ can be calculated in two ways. If $R(z)$ is written in the form (1.2), then

$$
\operatorname{deg}(R)=\max \{\operatorname{deg}(p), \operatorname{deg}(q)\} .
$$

Also, the degree of $R$ is the number (counted with multiplicity) of inverse images of any point of $\overline{\mathbf{C}}$. The Fatou-Julia theory applies to rational maps $R$ whose degree is at least two.

A dynamical system is formed by the repeated application (iteration) of the map $R$ from $\overline{\mathbf{C}}$ to itself. by

Definition. Given a point $z_{0} \in \overline{\mathbf{C}}$, the sequence $\left\{z_{n}\right\}$ is inductively defined

$$
z_{n+1}=R\left(z_{n}\right)
$$

This sequence is called the forward orbit of $z_{0}$ and is denoted $\mathrm{O}^{+}\left(z_{0}\right)$. 
There are many questions that one could ask about any given orbit $O^{+}\left(z_{0}\right)$. What are the limit points of $\mathrm{O}^{+}\left(z_{0}\right)$ ? What are the topological and analytic properties of the set of all limit points of $\mathrm{O}^{+}\left(z_{0}\right)$ ? What is the limiting distribution of $\mathrm{O}^{+}\left(z_{0}\right)$ ? How do the set of iterated preimages $\mathrm{O}^{-}\left(z_{0}\right)$ distribute themselves? In this paper we shall mostly confine our attention to topological questions.

An elementary way to distinguish different orbits is to count the number of points in the orbit.

DEFINITION. If $z_{n}=z_{0}$ for some $n$, then $z_{0}$ is a periodic point and $O^{+}\left(z_{0}\right)$ is a periodic orbit (often called a periodic cycle or simply a cycle). If $n$ is the first natural number such that $z_{n}=z_{0}$, then $n$ is called the period of the orbit. Usually, if the period of an orbit is one, we call $z_{0}$ a fixed point rather than a periodic point.

It is surprising how much of the theory in question is related to the distribution of periodic points. To illustrate the dynamical concepts we have just introduced, we make a few observations about the example $z \mapsto z^{2}$ which (in many ways) is the simplest example in the subject.

(1.3) EXAMPLE. Let $R(z)=z^{2}$. The behavior of the orbits depends upon where they lie relative to the unit circle $S^{1}$. The orbit of any point inside the circle approaches the origin (which is a fixed point), and any orbit outside the circle approaches $\infty$. Moreover, $R(\infty)=\infty$, so $\infty$ is another fixed point of the map $R$. Finally, to completely describe the dynamics of $R$, we need to describe the action of $R$ on the unit circle. But on the unit circle, $R\left(e^{i \theta}\right)=e^{i 2 \theta}, \theta \in \mathbf{R}$, so even though the map has a rich orbit structure, it is still a system which is amenable to computation. The reader who is unfamiliar with $R \mid S^{1}$ should try to describe this rich structure. It is not trivial.

Before we continue with our review, we should introduce the following notation, which can be the source of some confusion in this subject.

Notation. The symbol $R^{n}$ denotes the $n$-fold composition $R^{n}=R \circ R \circ$ .. $\circ R$ of the function $R$ with itself. Since we can multiply functions as well as compose them, we must be careful not to confuse $R^{2}$ with the function $S(z)=[R(z)]^{2}$.

In the theory of dynamical systems, the equivalence relation conjugation is often very useful. If $R(z)$ and $S(z)$ are two rational functions and $M(z)$ is a Möbius transformation ( $=$ bijective rational map) such that the diagram

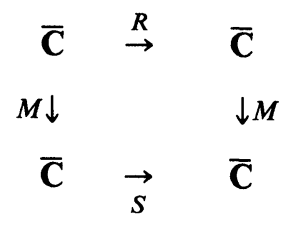

commutes, then $R$ and $S$ are (analytically) conjugate. Note that if $R$ and $S$ are conjugate by $M$, then $R^{n}$ and $S^{n}$ are also conjugate by $M$. So $R$ and $S$ are holomorphically the "same" dynamical system. Hence, the answer to any topological or conformal question about the system generated by $S$ can be 
obtained from the answer to the same question applied to $R$ by applying the $\operatorname{map} M$.

(1.4) EXAMPLE. To illustrate this notion, we consider an arbitrary quadratic polynomial $R(z)=a z^{2}+2 b z+d$. We can conjugate $R(z)$ to some quadratic polynomial $p(z)$ of the form $p(z)=z^{2}+c$. Let $M(z)=a z+b$ and $c=a d+$ $b-b^{2}$. Then we compute

$$
\begin{aligned}
M^{-1} \circ p \circ M(z) & =M^{-1}\left((a z+b)^{2}+c\right)=M^{-1}\left(a^{2} z^{2}+2 a b z+b^{2}+c\right) \\
& =\frac{\left(a^{2} z^{2}+2 a b z+b^{2}+c\right)-b}{a}=R(z) .
\end{aligned}
$$

As a result, we find that we need only study the class of quadratic polynomials of the form $z \mapsto z^{2}+c$ to understand the dynamics of all quadratic polynomials. Hence, the space of "all" dynamical systems of quadratic polynomials is really $\mathbf{C}$ rather than $\mathbf{C}^{3}$ as it might at first appear. Moreover, as we shall see in $\S 10$, there are technical reasons why this formulation is useful.

This is all we need to say in terms of background to the dynamical theory we will present. For convenience, we review a few essential concepts from complex analysis.

The notion of a normal family is closely connected with compactness of a set of analytic maps in the topology of uniform convergence on compact subsets. The following definition uses the spherical metric on $\overline{\mathbf{C}}$.

Definition. Let $U$ be an open subset of $\overline{\mathbf{C}}$ and $\mathscr{F}=\left\{f_{i} \mid i \in I\right\}$ a family of meromorphic functions defined on $U$ with values in $\overline{\mathbf{C}}$ ( $I$ is any index set). The family $\mathscr{F}$ is a normal family if every sequence $f_{n}$ contains a subsequence $f_{n_{j}}$ which converges uniformly on compact subsets of $U$.

It is awkward to relate this definition to any dynamical property, but Arzela's Theorem, relating normal families to equicontinuous ones, shows there are quite a few connections.

Definition. Let $X$ be a metric space with metric $d$. A family of functions $\left\{f_{i}: X \rightarrow X\right\}$ is an equicontinuous family if, given $\varepsilon>0$, there exists a $\delta>0$ such that $d\left(x_{1}, x_{2}\right)<\delta$ implies $d\left(f_{i}\left(x_{1}\right), f_{i}\left(x_{2}\right)\right)<\varepsilon$ for all $i$.

(1.5) TheOREM. The family $\left\{f_{i}: U \rightarrow \overline{\mathbf{C}}\right\}$ of meromorphic functions is a normal family if and only if it is an equicontinuous family on every compact subset of $U$.

Hence, normal families have values which do not diverge under iteration. In $\S 2$ we will be more precise about this connection.

In $\$ 6$ we shall also need the following sufficient condition for normality.

(1.6) THEOREM. Let $\left\{f_{i}: U \rightarrow \mathbf{C}\right\}$ be a holomorphic family of functions. If the family is locally uniformly bounded on $U$, then it is a normal family.

In particular, any family of holomorphic mappings to the unit disk is normal. The reader is referred to Ahlfors' book [A1] for a proof of both of these theorems and a more complete discussion of normal families. 
We recall the elementary version of Schwarz's lemma.

Notation. Given $z \in \mathbf{C}$ and $r>0$, we denote the open disk centered at $z$ of radius $r$ (in the Euclidean metric) by $D_{r}(z)$, and the symbol $D_{r}$ will represent the same set as $D_{r}(0)$.

(1.7) THEOREM. Let $f: D_{1} \rightarrow D_{1}$ be an analytic function such that $f(0)=0$. Then

(a) $|f(z)| \leqslant|z|$ for all $z \in D_{1}$ and

(b) $\left|f^{\prime}(0)\right| \leqslant 1$.

If equality holds in either (a) for any $z \in D_{1}-\{0\}$ or in (b), then $f(z)=e^{i \theta} z$ where $\boldsymbol{\theta} \in \mathbf{R}$.

On the whole, we shall not need much of the theory of Riemann surfaces. However, in \$3, we use the uniformization theorem-the cornerstone of the classification of Riemann surfaces. Recall that a Riemann surface is a one (complex) dimensional manifold. The uniformization theorem classifies all simply connected Riemann surfaces.

(1.8) THEOREM. Every simply connected Riemann surface is conformally equivalent to either $\overline{\mathbf{C}}, D_{1}$, or $\mathbf{C}$.

In our context we will only encounter Riemann surfaces which are open subsets of $\overline{\mathbf{C}}$ or which are $\mathbf{C} / L$, where $L$ is a lattice. The reader should use the uniformization theorem to show that if $U$ is a domain in $\mathbf{C}$ which has at least three boundary points, then its universal cover $U$ is conformally equivalent to $D_{1}$.

There are many good references on the subject of Riemann surfaces. Abikoff [Ab1, Ab2], Ahlfors [A2], and Farkas and Kra [FK] are particularly appropriate for the uniformization theorem.

2. The dynamical dichotomy of Fatou and Julia. Fatou and Julia studied the iteration of rational maps $R: \overline{\mathbf{C}} \rightarrow \overline{\mathbf{C}}$ under the assumption that $\operatorname{deg}(R) \geqslant 2$. Basically they focused on a disjoint invariant decomposition of $\overline{\mathbf{C}}$ into two sets. One of these sets is often called the Julia set. The other set does not have a standard name, and in this paper, we shall refer to it as the Fatou set.

Definition. A point $z \in \overline{\mathbf{C}}$ is an element of the Fatou set $F(R)$ of $R$ if there exists a neighborhood $U$ of $z$ in $\overline{\mathbf{C}}$ such that the family of iterates $\left\{R^{n} \mid U\right\}$ is a normal family. The Julia set $J(R)$ is the complement of the Fatou set.

The classical papers denote the Julia set by $\mathscr{F}$, but since the term "Julia set" is now commonly used, we shall denote it by $J(R)$. The Fatou set did not have an explicit name classically, and it is now sometimes referred to as the domain of equicontinuity. Often we use $F$ and $J$ if there is no confusion about the map.

In this section we shall prove that the Julia set is always nonempty, but the reader should beware that the Fatou set can be void. In $\$ 3$ we will show that the Julia set of the map $z \mapsto\left(z^{2}+1\right)^{2} / 4 z\left(z^{2}-1\right)$ is the entire Riemann Sphere (this is Lattès' example-see (3.2)).

The Fatou set is open by definition, and since $R$ is both continuous and an open mapping, proving that $F$ is completely invariant is straightforward. In 
other words, if $z \in F$ then $R(z) \in F$ and $R^{-1}(z) \subset F$. Consequently, the Julia set is also completely invariant and compact.

If we consider the example $R(z)=z^{2}$ again and take a point $z_{0}$ inside the unit circle, then there exists an open disk $U$ around $z_{0}$ on which the sequence $R^{n} \mid U$ converges to the constant function $C(z)=0$ for all $z \in U$. Hence, the interior of the unit circle is a subset of the Fatou set. Likewise, the exterior is also a subset of the Fatou set although the limit function is different. The unit circle is, however, equal to the Julia set because the family $\left\{R^{n}\right\}$ is not equicontinuous on any open set which intersects the circle.

The Julia set of the map $S(z)=z^{2}-2$ is also simple to describe. It is the interval $[-2,2]$ on the real line. This is not so easy to see from the definitions, but the reader may enjoy investigating this example before we prove this later (see (7.12(1))).

The above two examples are deceiving. In general, Julia sets are not smooth. Figures (2.1)-(2.5) illustrate the types of complexity that Julia sets usually possess.

The Julia set in Figure (2.1) is a fractal (see Mandelbrot [M1]). In fact, most Julia sets are fractals.

Figures (2.1)-(2.5) are neither the most accurate nor the most elaborate ones available. There are many methods with which one can draw Julia sets, and the trade-offs are (as usual) quality versus computer power and time. In $\$ 3$ we shall encounter situations where the more expensive methods seem (at our present state of knowledge) absolutely necessary. But for our purposes, the accuracy of these pictures shall suffice. The reader should, however, seek out some of the more intricate pictures which have been published recently and which will be in forthcoming publications. This author is aware of the articles of Curry, Garnett, and Sullivan [CGS], Douady [D], Douady and Hubbard [DH], and Mandelbrot [M1, M2], and this is probably a very incomplete list of all such publications.

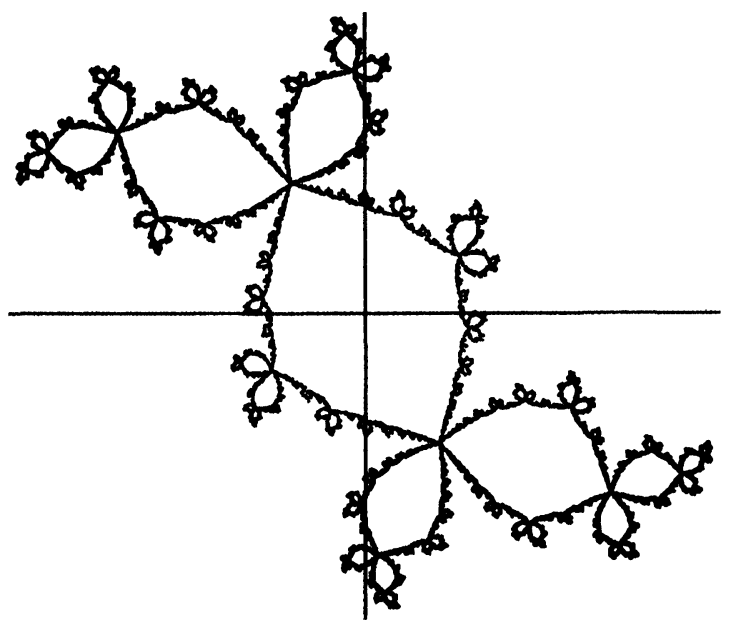

(2.1) Figure. Douady's Rabbit. The Julia set of the map $z \mapsto z^{2}+c$, where $c$ satisfies $c^{3}+2 c^{2}+$ $c+1=0$ and $\operatorname{Im}(c)>0$. The approximation $c \approx-0.12256117+0.74486177$ was used to generate this figure. This Julia set is connected, and the Fatou set consists of infinitely many, simply connected domains. 


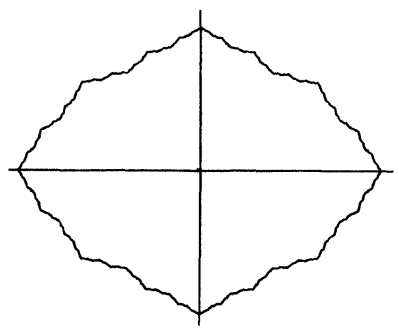

(2.2) FIgURE. This is the Julia set of the quadratic polynomial $p(z)=z^{2}-0.3125$. It is a Jordan curve. In addition, Sullivan [S4] has shown that it is a quasi-circle (the image of the unit circle under a quasi-conformal homeomorphism-see Ahlfors [A3]) whose Hausdorff dimension is greater than one. Ruelle's results [R] on the real analytic nature of Hausdorff dimension are also relevant.

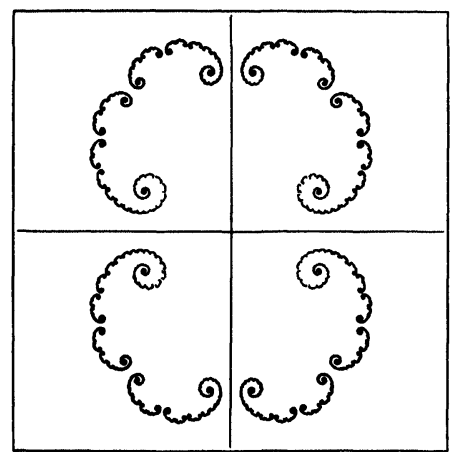

(2.3) FIGURE. The Julia set of $p(z)=z^{2}+.3$. Theorem (9.9) applies to this map; therefore, $J(p)$ is a Cantor set. The next figure shows some of the finer detail.

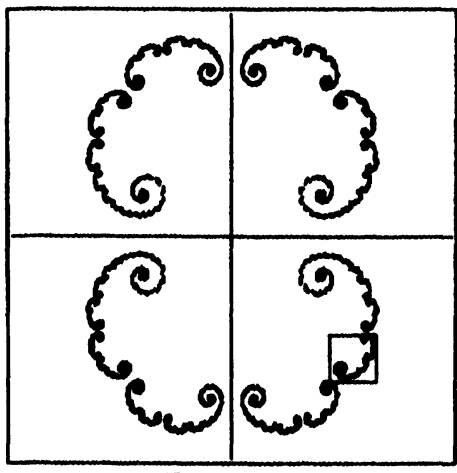

FIGURE a

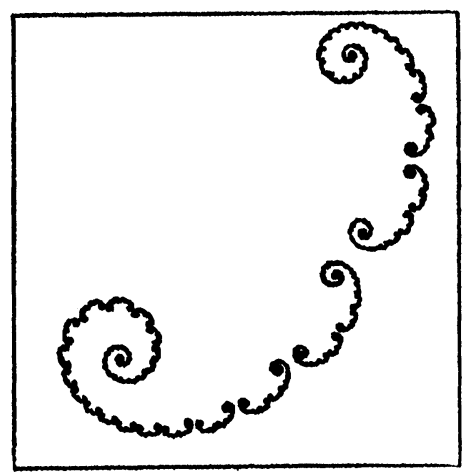

FIgURE $b$

(2.4) Figures. These figures illustrate the fractal nature of the Julia set in the previous figure. Figure $b$ is an enlargement of the small box in Figure $a$.

We conclude our introduction to the dichotomy with the next theorem.

(2.6) THEOREM. The Julia set is nonempty.

Proof. Suppose $J(R)=\varnothing$. Then $F(R)=\overline{\mathbf{C}}$ and, therefore, the family $\left\{R^{n}\right\}$ is normal on $\overline{\mathbf{C}}$. A convergent subsequence $R^{n_{i}}$ converges uniformly (in 


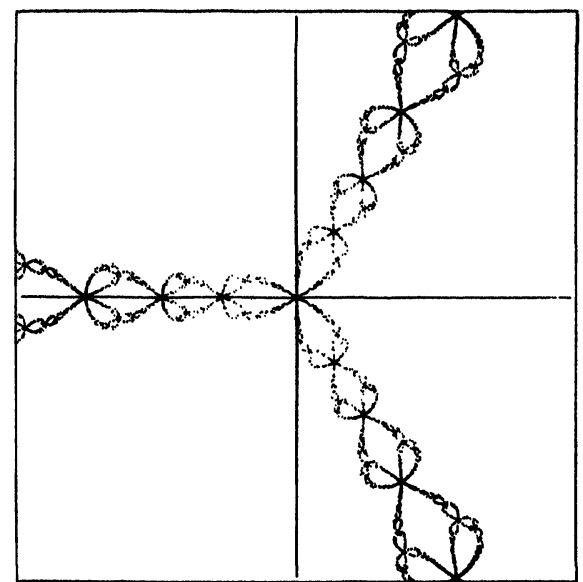

(2.5) FIGURE. Newton's method applied to the polynomial equation $z^{3}-1=0$ yields the rational function $N(z)=\left(2 z^{3}+1\right) / 3 z^{2}$. The orbit of almost any initial value will converge to a root of the equation. The Julia set is the set of initial values for which the method fails.

the spherical metric) to a meromorphic limit function $S$. Since $S$ is a continuous function of the two-sphere, we can now derive a contradiction by considering the topological degree of $S$.

$$
\operatorname{deg}(S) \leftarrow \operatorname{deg}\left(R^{n_{i}}\right) \rightarrow \infty \quad \text { as } n_{i} \rightarrow \infty
$$

But $\operatorname{deg}(S)$ is finite.

3. Periodic points. In this section we discuss the dynamics in a neighborhood of a periodic point. In particular, we give conditions which often determine in which set, $F$ or $J$, the periodic point lies.

Definition. Let $z_{0}$ be a periodic point of period $n$. Then the number $\lambda_{z_{0}}=\left(R^{n}\right)^{\prime}\left(z_{0}\right)$ is the eigenvalue of the periodic orbit.

Of course, the chain rule implies that $\lambda_{z_{0}}$ is the product of the derivatives of the map $R$ along the orbit. Consequently, $\lambda_{z_{0}}$ is an invariant of the orbit $\mathrm{O}^{+}\left(z_{0}\right)$ rather than the particular point $z_{0}$. Whenever we discuss just one periodic orbit, we drop the subscripts and simply denote the eigenvalue by $\lambda$.

Definition. A periodic orbit $O^{+}\left(z_{0}\right)$ is:

(1) attracting if $0<|\lambda|<1$,

(2) superattracting if $\lambda=0$,

(3) repelling if $|\lambda|>1$, or

(4) neutral if $|\lambda|=1$.

A. Attracting and repelling periodic points. Using the Mean Value Theorem and Arzela's Theorem ((1.5)), it is easy to prove

(3.1) Proposition. If $\mathrm{O}^{+}\left(z_{0}\right)$ is a (super)attracting periodic orbit, then it is contained in $F$. If it is a repelling periodic orbit, then it is contained in $J$. 
At this point it may be worthwhile for the reader to go back to the example $z \mapsto z^{2}$ and check the proposition with our original analysis of the Julia set.

Definition. A point $z$ is eventually periodic if, for some $n, R^{n}(z)$ is a periodic point. The point $z$ is preperiodic if it is eventually periodic but not periodic.

(3.2) LATtès' ExAmples [L]. We can now sketch why $J(R)=\overline{\mathbf{C}}$ for the map $R(z)=\left(z^{2}+1\right)^{2} / 4 z\left(z^{2}-1\right)$. Let $L$ be any discrete lattice in $\mathbf{C}$ consisting of all $n_{1} w_{1}+n_{2} w_{2}$, with $n_{i} \in \mathbf{Z}$, where $w_{1}$ and $w_{2}$ are fixed complex numbers such that $w_{2} / w_{1} \notin \mathbf{R}$. First, note that $L$ is invariant by multiplication by an integer, so any such multiplication induces an endomorphism of the complex torus $T^{2}=\mathrm{C} / L$. Secondly, the Weierstrass $\wp$-function

$$
\wp(z)=\frac{1}{z^{2}}+\sum_{\substack{w \in L \\ w \neq 0}}\left(\frac{1}{(z-w)^{2}}-\frac{1}{z^{2}}\right)
$$

can be thought of as a function from $T^{2}$ to $\overline{\mathbf{C}}$ (see [A1 and Co]). Since $M$ preserves the inverse images of the map $\wp: T^{2} \rightarrow \overline{\mathbf{C}}$, we get a rational map $R_{L, M}$ which satisfies the following commutative diagram:

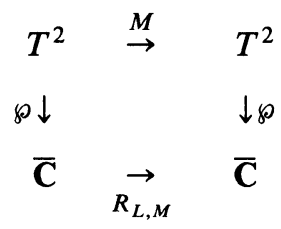

To show that $J\left(R_{M, L}\right)=\overline{\mathbf{C}}$ whenever $\operatorname{deg}(M) \geqslant 2$, we exhibit a dense subset of $\overline{\mathbf{C}}$ of eventually periodic points which are all in $J\left(R_{M, L}\right)$. Suppose $z=q_{1} w_{1}$ $+q_{2} w_{2}$ with $q_{i} \in \mathbf{Q}(i=1,2)$. Then the point $\ell(z)$ in $\overline{\mathbf{C}}$ is eventually periodic under the map $R_{L, M}$. In fact, if

$$
\begin{aligned}
A_{N}=\{z \in \mathbf{C} \mid z & =\left(a_{1} / b_{1}\right) w_{1}+\left(a_{2} / b_{2}\right) w_{2} \\
& \text { where } \left.a_{i}, b_{i} \in \mathbf{Z} \text { and } b_{i} \leqslant N(i=1,2)\right\},
\end{aligned}
$$

and if $B_{N}$ is the finite subset of $T^{2}$ defined by $B_{N}=A_{N} / L$, then $M\left(B_{N}\right) \subset B_{N}$, and every point of $B_{N}$ must be eventually periodic. Moreover, the point $\ell(z)$ belongs to $J\left(R_{L, M}\right)$ because the norm of any eigenvalue of any periodic point of $M$ is greater than one when $\operatorname{deg}(M) \geqslant 2$.

The explicit formula above results from choosing $M$ to be multiplication by 2. See Lattès [L] or Herman [H1] for a derivation of the above rational function.

We know everything about the local dynamics in the attracting case and everything about the behavior of successive images until they leave a neighborhood of the repelling orbit. The map $R^{n}$ is locally conjugate to its derivative.

(3.3) THEOREM. Let $z_{0}$ belong to an attracting periodic orbit of period $n$. There exists a neighborhood $U$ of $z_{0}$ and a unique analytic homeomorphism $\phi: U \rightarrow D_{r}$ 
(for some $r$ ) such that $\phi\left(z_{0}\right)=0, \phi^{\prime}\left(z_{0}\right)=1$ and the diagram

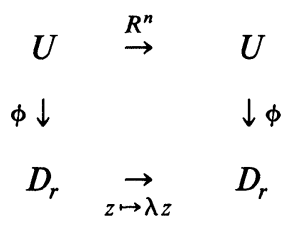

commutes.

From this theorem we see that the neighborhood $U$ is forward invariant, i.e. $R^{n}(U) \subset U$, and the orbit of every point in $U$ is asymptotic to the periodic orbit $O^{+}\left(z_{0}\right)$.

There are both analytic and geometric proofs of the existence of $\phi$. The oldest proof is due to Koenigs in the nineteenth century. Basically he proved that the sequence of functions $\left(R^{k n}(z)-z_{0}\right) / \lambda^{k}$ tends uniformly to a holomorphic function $\phi$. Then it is easy to verify that $\phi \circ R^{n}(z)=\lambda(\phi(z))$. The reader is referred to Siegel and Moser [SM, p. 188] for the technical details.

Theorem (3.3) also yields a local conjugacy in the repelling case. If $\mathrm{O}^{+}\left(z_{0}\right)$ is a repelling periodic orbit, then (3.3) can be applied to the inverse of $R^{n}$ which has $\mathrm{O}^{+}\left(z_{0}\right)$ as an attracting periodic orbit. However, we get less information because the orbit of every point $z \in U$, except $z_{0}$, eventually leaves the neighborhood $U$.

Finally, it is worth noting that $R$ need only be analytic in (3.3).

B. The superattracting case. Superattracting periodic orbits are also locally conjugate to simple maps. Yet the dynamics of these maps is much more interesting.

The following theorem applies to any analytic function-not just a rational function. We use the notation $\left(R^{n}\right)^{(k)}\left(z_{0}\right)$ to represent the $k$ th derivative of $R^{n}$.

(3.4) TheOREM. Let $O^{+}\left(z_{0}\right)$ be a superattracting periodic orbit. Suppose $k \geqslant 2$, $\left(R^{n}\right)^{(k)}\left(z_{0}\right) \neq 0$, and

$$
\left(R^{n}\right)^{\prime}\left(z_{0}\right)=\left(R^{n}\right)^{(2)}\left(z_{0}\right)=\cdots=\left(R^{n}\right)^{(k-1)}\left(z_{0}\right)=0 .
$$

Then there exists a neighborhood $U$ of $z_{0}$ and an analytic homeomorphism $\phi: U \rightarrow D_{r}$ (for some $r$ ) such that $\phi\left(z_{0}\right)=0, \phi^{\prime}\left(z_{0}\right)=1$, and the following diagram commutes:

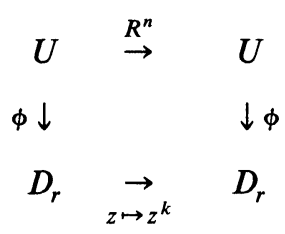

Again we have a forward invariant neighborhood, and every orbit in the neighborhood is asymptotic to $O^{+}\left(z_{0}\right)$. But in this case, the map $z \mapsto z^{k}$ is not locally invertible. This gives us a great deal of information which will be particularly helpful in the analysis of the dynamics of polynomials. 
This theorem is not as commonly known; consequently, we include the proof from Fatou [F1, pp. 187-189]. He credits Boettcher [Bo] as the first person to demonstrate the existence of the conjugacy.

Before giving the proof, we recall a few facts about fractional exponents. The map $z \mapsto z^{k}$ is a branched cover of $\mathbf{C}$ by itself. If we have a map $S: D_{r} \rightarrow \mathrm{C}$, we can often find a map, which we denote $(S(z))^{1 / k}$, such that the following diagram commutes:

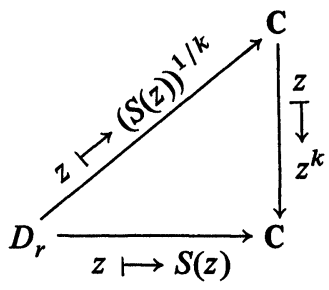

For example, if $0 \notin S\left(D_{r}\right)$, then covering space theory gives us $k$ maps which work. Also, if $S(0)=0$ and $S(z) \neq 0$ for all $z \in D_{r}-\{0\}$, and if the local degree of $S$ around 0 is a multiple of $k$, then there are also $k$ maps that will work. The exponential notation is used in order to summarize a few properties of these maps such as $(S(z))^{1 / 2} /(S(z))^{1 / 4}=(S(z))^{1 / 4}$. Unfortunately we have chosen to use exponents in two different ways. Hence, $\left(S^{n}(z)\right)^{1 / n} \neq S(z)$.

The idea of the following proof of (3.4) is exactly the same as Koenig's proof. A sequence $\left\{\phi_{n}\right\}$ of maps is produced by iterating the rational function $n$ times and then taking the appropriate inverse of $z \mapsto z^{k^{n}}$. This sequence converges to the conjugacy.

ProOF OF (3.4). We prove the theorem in the case where $n=1$ and $z_{0}=0$.

The power series expansion of $R(z)$ is $a_{k} z^{k}+a_{k+1} z^{k+1}+\cdots$. If we conjugate $R(z)$ by the map $z \mapsto b z$, where $b^{k-1}=\left(a_{k}\right)^{-1}$, we get the map

$$
S(z)=z^{k}+b_{k+1} z^{k+1}+\cdots .
$$

Proving the result for $S(z)$ will suffice.

We always work inside a disk $D_{r}$ such that $S\left(D_{r}\right) \subset \operatorname{int}\left(D_{r}\right)$ and

$$
\lim _{n \rightarrow \infty} S^{n}(z)=0 \quad \text { for all } z \in D_{r} \text {. }
$$

We define

$$
\phi_{n}(z)=\left(S^{n}(z)\right)^{1 / k^{n}},
$$

choosing $\phi_{n}^{\prime}(0)=1$. If we prove that the sequence $\left\{\phi_{n}\right\}$ converges uniformly to a map $\phi$, we have the desired conjugacy because

$$
\phi \circ S=\lim _{n \rightarrow \infty}\left[S^{n+1}(z)\right]^{1 / k^{n}}
$$

and

$$
[\phi(z)]^{k}=\lim _{n \rightarrow \infty}\left[\left(S^{n+1}(z)\right)^{1 / k^{n+1}}\right]=\phi \circ S .
$$


To prove that $\left\{\phi_{n}\right\}$ converges uniformly on some neighborhood of 0 , we introduce the function

$$
H(z)=\phi_{1}(z) / z .
$$

Note that $H(0)=1$. Using this function we see that

$$
\frac{\phi_{n+1}(z)}{\phi_{n}(z)}=\left[\frac{\phi_{1}\left(S^{n}(z)\right)}{S^{n}(z)}\right]^{1 / k^{n}}=\left[H\left(S^{n}(z)\right)\right]^{1 / k^{n}} \text {. }
$$

Using the notation $\phi_{0}(z)=z$, we have

$$
\phi_{n+1}(z)=z \prod_{i=0}^{n} \frac{\phi_{i+1}(z)}{\phi_{i}(z)}=z \prod_{i=0}^{n}\left[H\left[S^{n}(z)\right]\right]^{1 / k^{n}} .
$$

The uniform convergence of $\left\{\phi_{n}\right\}$ can now be established by proving that the infinite product converges uniformly. This, in turn, is established by taking the logarithm of the infinite product (using the principal branch). We get the infinite series

$$
\sum_{n=0}^{\infty} \frac{1}{k^{n}} \log \left(H\left(S^{n}(z)\right)\right) .
$$

Using the above-mentioned dynamical properties of $S$ in $D_{r}$, we know that $\left|H\left(S^{n}(z)\right)-1\right|<1 / 2$ and $\left|H\left(S^{n}(z)\right)-1\right| \leqslant C\left|S^{n}(z)\right|$ for some constant $C$. If $|\alpha|<1 / 2$,

$$
|\log (1+\alpha)| \leqslant|\alpha|+|\alpha|^{2}+\cdots \leqslant 2|\alpha|
$$

so

$$
\left|\log H\left(S^{n}(z)\right)\right| \leqslant 2 C\left|S^{n}(z)\right| .
$$

Rather than (3.5), we consider the series

$$
2 C \sum_{n=0}^{\infty} \frac{\left|S^{n}(z)\right|}{k^{n}} \leqslant 2 C \sum_{n=0}^{\infty} \frac{r}{k^{n}},
$$

which clearly converges.

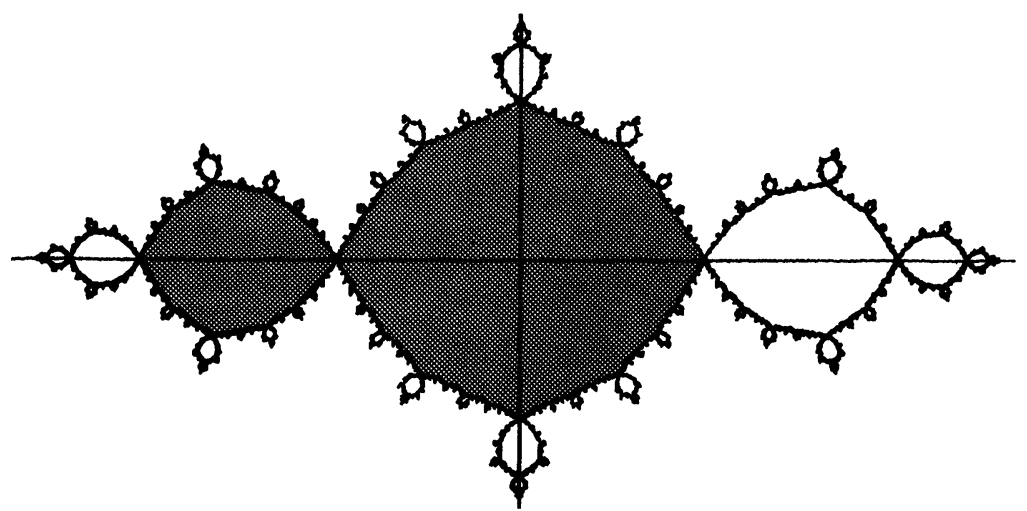

(3.7) FIGURE. The Julia set of $z \mapsto z^{2}-1$. The two shaded components of the Fatou set are mapped one to the other. 
(3.6) REMARK. Douady's Rabbit (Figure (2.1)) has a superattractive period 3 point at the origin. In fact, the choice of $c$ was made so that the origin (which is the critical point) is a periodic point of period 3.

Figure (3.7) has a period 2 superattracting orbit contained in the shaded regions of the Fatou set.

C. Neutral orbits. The local dynamics in a neighborhood of a neutral periodic orbit is not so easy to describe. In fact, as we shall see, there are open questions relating to this case.

Again it is enough to describe the situation where $z_{0}$ is a fixed point. So we assume $R\left(z_{0}\right)=z_{0}$ and $|\lambda|=\left|R\left(z_{0}\right)\right|=1$. If we try to conjugate $R \mid U$ to its derivative, then the diagram

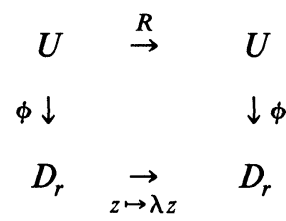

yields a functional equation

$$
\phi \circ R(z)=\lambda(\phi(z))
$$

This equation is called the Schröder Functional Equation. Fatou related solutions $\phi$ of (SFE) to membership in the Fatou set. Geometrically this equation means that $z_{0}$ is the center of a disk on which the map is a rotation (see (7.3(4))).

(3.8) ThEOREM. Let $z_{0}$ be a neutral fixed point. Then $z_{0} \in F$ if and only if (SFE) has an analytic solution in some neighborhood of $z_{0}$.

Obviously the same statement holds for a neutral periodic orbit of period $n$ once $R$ is replaced by $R^{n}$ in (SFE). We prove this theorem here, although it is logically out of order. In the proof we shall use the fact that the Julia set contains at least three points. In fact, it is always uncountable because it is always a perfect set (see (4.8)).

PROOF. If (SFE) has a solution, then membership of $z_{0}$ in $F$ can be verified directly from the definitions.

Suppose $z_{0} \in F$ and let $U$ be the maximal domain such that $z_{0} \in U$ and $U \subset F$. Since $U \cap J=\varnothing, U$ misses three points and the uniformization theorem ((1.8)) implies that the universal cover $\tilde{U}$ of $U$ is conformally equivalent to the unit disk $D_{1}$. We choose a cover $p: D_{1} \rightarrow U$ such that $p(0)=z_{0}$ and we choose a lift $\tilde{R}$ of $R / U$ so that $\tilde{R}(0)=0$. Then $\tilde{R}: D_{1} \rightarrow D_{1}$ and $\left|(\tilde{R})^{\prime}(0)\right|=1$. An application of Schwarz's lemma (1.7) yields the fact that $\tilde{R}(z)=\lambda z$. The universal cover $p$ is the solution $\phi$ to the equation (SFE).

REMARKS. (1) Note that the same proof can be used to prove that the existence of a topological conjugacy implies the existence of an analytic conjugacy.

(2) Another proof which does not use the uniformization theorem or the fact that $J$ is uncountable was shown to me by P. Collet, R. de la Llave, and O. Lanford. Let 


$$
\Psi_{n}(z)=\frac{1}{n}\left(\sum_{p=0}^{n-1} \lambda^{-p} R^{p}(z)\right) .
$$

If the sequence $\left\{R^{p}\right\}$ is normal around $z_{0}$, the $\Psi_{n}$ converge to a map $\Psi$ such that $\Psi_{n} \circ R \rightarrow \lambda \Psi$.

An easy consequence of (3.8) concerns the case where $\lambda$ is a root of unity.

(3.9) COROLLARY. If $\lambda$ is a root of unity, then (SFE) does not have a solution.

PROof. Replacing $R$ by some iterate if necessary, we may assume $z_{0}$ is a fixed point. Suppose $\lambda^{l}=1$ and (SFE) has a solution in a neighborhood $U$ of $z_{0}$. Then $\phi \circ R^{l} \circ \phi^{-1}=\mathrm{Id}$, and therefore $R^{l}=\mathrm{Id}$ on $U$. Since $R$ is analytic, it must be the identity on $\overline{\mathbf{C}}$, contradicting our assumption that the degree of $R$ is greater than one.

Both Fatou [F1, pp. 191-221] and Julia [J, pp. 297-311] extensively discussed the root of unity case, and they both credit Leau (Thèse, 1897) with the initial analysis of the $\lambda=1$ case. More recently, Camacho [Ca] has classified the dynamics up to topological conjugacy. Without proof, we give a description of some of their results.

First we state the topological result.

(3.10) THEOREM. Let $f(z)=\lambda z+a_{2} z^{2}+a_{3} z^{3}+\cdots$ be an analytic map in the neighborhood of the origin. Suppose $\lambda^{n}=1$ and $\lambda^{m} \neq 1$ for $1<m<n$. Then either $f^{n}=\mathrm{Id}$ or there is a local homeomorphism $h$ and an integer $k>1$ such that $h(0)=0$ and $h \circ f \circ h^{-1}(z)=\lambda z\left(1+z^{k n}\right)$.

In order to understand what this means, it is necessary to analyze the dynamics of the map $z \mapsto \lambda z\left(1+z^{k n}\right)$. The condition $z^{k n} \in \mathbf{R}$ describes a set of lines through the origin. Note that the map $g(z)=z\left(1+z^{k n}\right)$ leaves these lines invariant. Then $z \mapsto \lambda z\left(1+z^{k n}\right)$ is the composition of $g$ with the periodic rotation $z \mapsto \lambda z$. The dynamics of the standard form $g$ in the cases $k=1,2$, and 3 are illustrated in Figures (3.11a)-(3.11c).

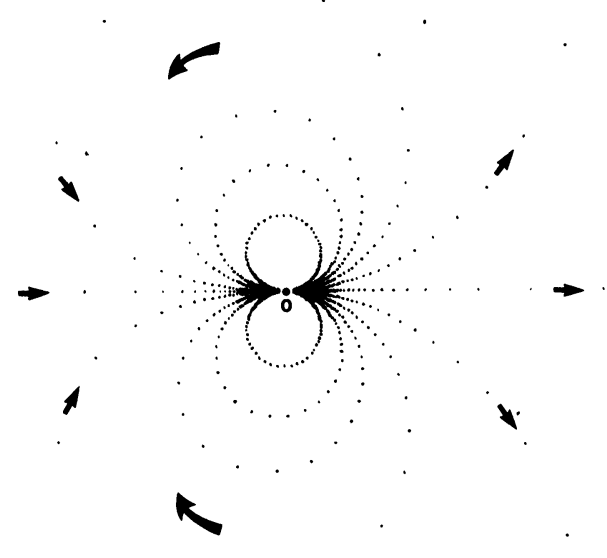

(3.11a) FIGURE. 13 partial orbits of the map $z \mapsto z(1+z)$. 


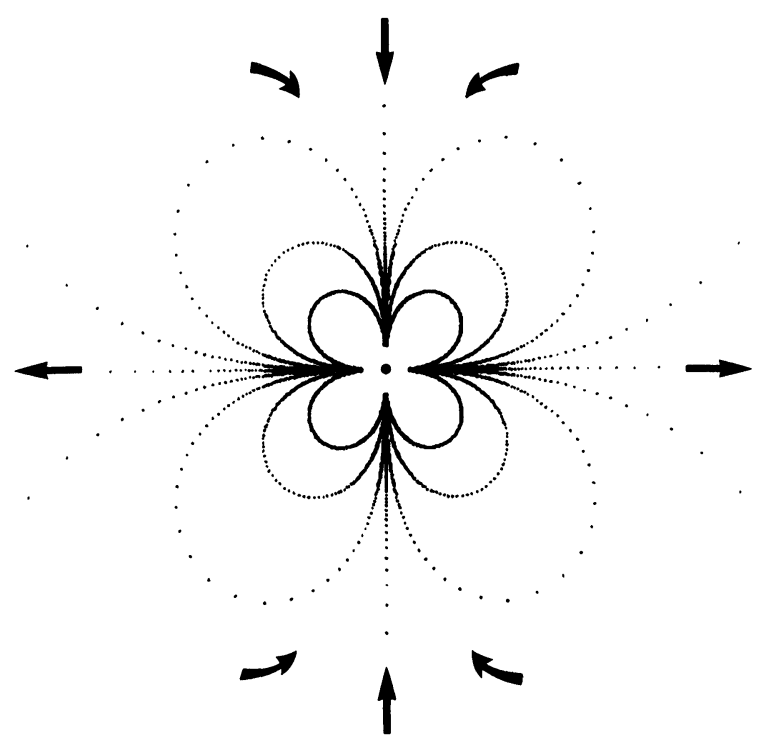

(3.11b) FIGURE. 21 partial orbits of the map $z \mapsto z\left(1+z^{2}\right)$.

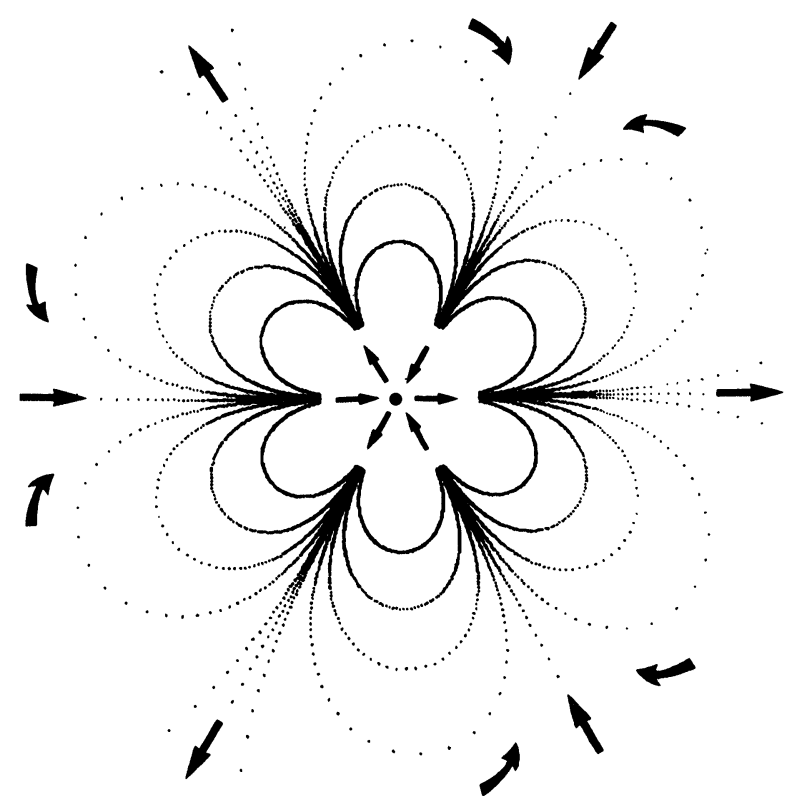

(3.11c) FigURE. 37 partial orbits of the map $z \mapsto z\left(1+z^{3}\right)$.

Using techniques similar to Camacho, Fatou and Julia also obtained some analytic information. These results are usually referred to as the Flower Theorem.

(3.12) THEOREM. Let $f(z)=\lambda z+a_{2} z^{2}+\cdots$ be an analytic map in a neighborhood of the origin and suppose $\lambda^{n}=1$ and $\lambda^{m} \neq 1$ for $1<m<n$. If $f^{n} \neq \mathrm{Id}$, there is an integer $k$ and $n k$ analytic curves which are pairwise tangent 
at the origin and which bound petals. The union of the petals is forward invariant, and any orbit in a petal is asymptotic to the origin. Any compact set inside the petal converges uniformly to the origin under $f^{n}$.

These petals do not contain all the orbits which are asymptotic to the origin because that set does not have an analytic boundary. In the rational case, that set is part of the Julia set, and the Julia set does not have tangents. Figures (3.13)-(3.16) illustrate the relationship between the Julia set and these petals. The computer points are the Julia set, and the petals are hand-drawn and shaded. In $\$ \$ 4$ and 5 we shall see that the Julia set must contain a sequence of repelling periodic points which, in this case, converge to the origin. These points approach the origin between the petals. Figures (3.13)-(3.15) relate exactly to the pictures in Figures (3.11a)-(3.11c).

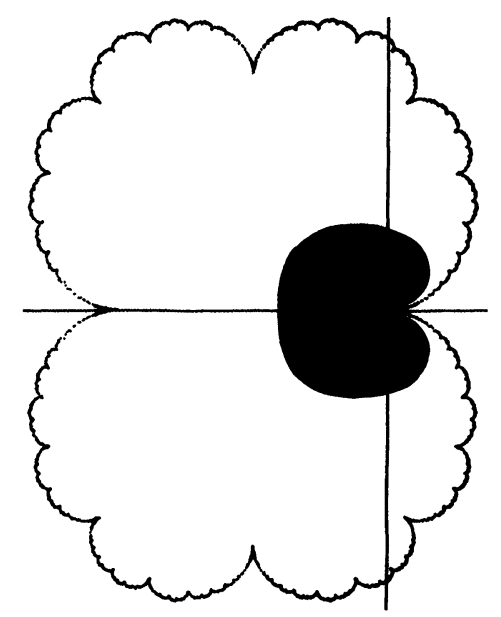

(3.13) Figure. The Julia set of $z \mapsto z+z^{2}$ and the associated petal. In this case the petal is cardioid shaped.

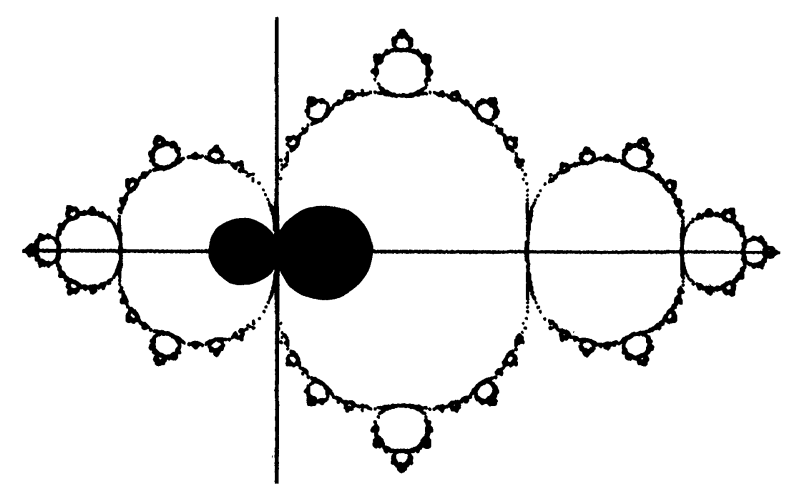

(3.14) FIGURE. The Julia set of $z \rightarrow-z+z^{2}$ and the petals of the origin. 


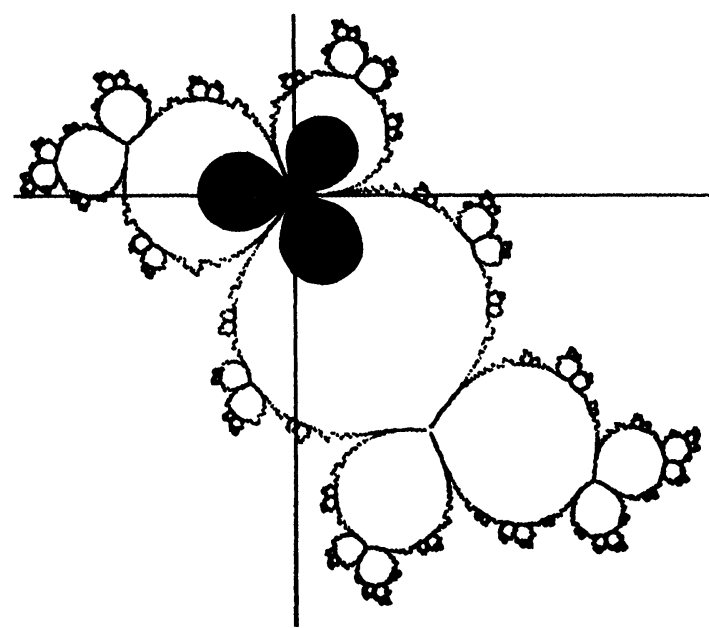

(3.15) FIGURE. The Julia set of $z \mapsto e^{2 \pi i / 3} z+z^{2}$ and its petals. Compare this figure with Douady's Rabbit (Figure (2.1)).

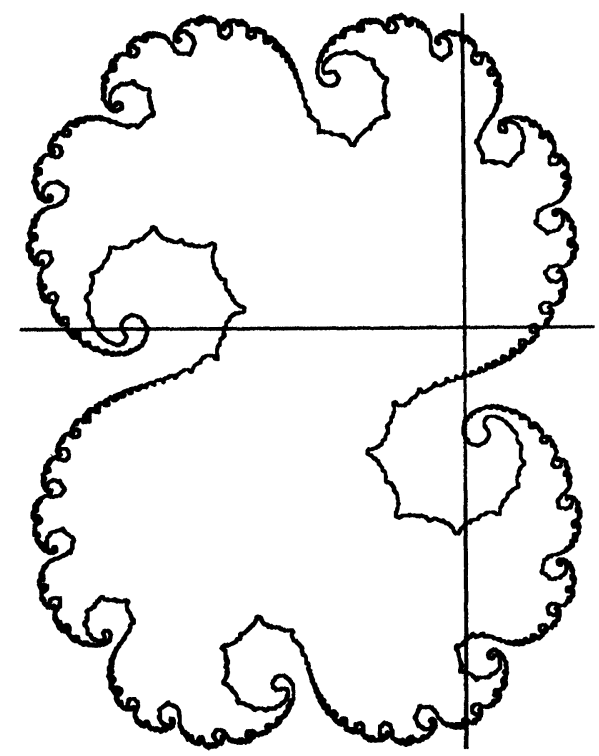

(3.16) FIGURE. The Julia set of $z \mapsto e^{2 \pi i / 20} z+z^{2}$. Here the Julia set should approach the origin in twenty different directions (between twenty petals), yet it is hard to get a good picture of this behavior. We include the picture to inspire the reader to imagine how the Julia set of $R_{\lambda}(z)=\lambda z$ $+z^{2}$, where $|\lambda|=1$, varies with $\lambda$ as $\lambda$ approaches 1 . Also the reader should be inspired to derive a good algorithm for plotting the Julia set in this case.

There were two major questions left unresolved by Fatou and Julia.

(3.17) Does (SFE) ever have a solution?

(3.18) If so, when?

In 1942, C. Siegel [Si] found a subset $\Lambda$ of the unit circle of full measure such that, whenever the eigenvalue is in $\Lambda$, then (SFE) does have a solution. 
(3.19) THEOREM. Let $\lambda_{z_{0}}=e^{2 \pi i \alpha}$ where $\alpha \in \mathbf{R}-\mathbf{Q}$. Suppose there exist positive constants $a$ and $b$ such that $|\alpha-p / q|>a / q^{b}$ for all $p, q \in \mathbf{Z}$ with $q \geqslant 1$. Then the Schröder Functional Equation does have a solution.

This condition is satisfied for a set of $\lambda_{z_{0}}$ of full measure in the unit circle.

The original proof $[\mathbf{S i}]$ is an extremely difficult computation involving delicate number theory. A different proof, which uses a version of Newton's method applied to function spaces, is contained in Siegel and Moser [SM], and recently a relatively simple proof for a smaller class of numbers using techniques of M. Herman has been circulated (see [dL and H1]).

Rather than discuss the proof, we recall some facts about diophantine approximation and continued fraction expansions which explain the number theory condition in the hypothesis. This condition loosely says " $\alpha$ is badly approximated by rational numbers". If we write $\alpha$ in its continued fraction expansion, we can make this statement precise. Let

$$
\alpha=a_{0}+\frac{1}{a_{1}+\frac{1}{a_{2}+\frac{1}{a_{3}+\ddots}}}
$$

and define the convergents $p_{n} / q_{n} \in \mathbf{Q}$ of $\alpha$ by

$$
\frac{p_{n}}{q_{n}}=a_{0}+\frac{1}{a_{1}+\frac{1}{a_{2}+\cdot \frac{1}{a_{n}}}}
$$

They are the best rational approximants to $\alpha$ (see Niven [Ni]), and the accuracy of the approximation is given by the inequality

$$
\frac{1}{\left(a_{n+1}+2\right) q_{n}^{2}}<\left|\alpha-\frac{p_{n}}{q_{n}}\right|<\frac{1}{a_{n+1} q_{n}^{2}} .
$$

For example, if the terms $a_{i}$ stay bounded, we can verify the hypothesis of Siegel's Theorem. Using the mean value theorem, it is easy to show that any algebraic number of degree 2 has such a continued fraction expansion. Moreover, the same argument also shows that the hypothesis of (3.19) is satisfied for an algebraic number of degree $n$ with $b=n+1$ (Niven [Ni]). So all algebraic numbers are in $\Lambda$. But as mentioned above, the set $\Lambda$ contains many other points because it is a set of full measure (Siegel and Moser [SM, p. 190]).

We have seen that if $z_{0}$ is a fixed point with derivative $\lambda_{z_{0}}$, where $\lambda_{z_{0}}=e^{2 \pi i \alpha}$, then $\alpha \in \mathbf{Q}$ implies $z_{0} \in J$, and $\alpha \in \Lambda$ implies $z_{0} \in F$. It would be nice to believe that $z_{0} \in F$ whenever $\alpha \notin \mathbf{Q}$. However, the following example of Cremer $[\mathbf{C}]$ shows that things are not this simple. 
(3.21) EXAMPLE. Let $p(z)=z^{d}+\cdots+\lambda z$. Suppose $\lambda=e^{2 \pi i \alpha}$, where $\alpha \in$ $\mathbf{R}-\mathbf{Q}$, and $\left|\lambda^{n}-1\right| \leqslant(1 / n)^{d^{n}-1}$ for infinitely many natural numbers $n$. Since the periodic points satisfy $p^{n}(z)=z$, we can calculate them by solving

$$
z^{d^{n}}+\cdots+\lambda^{n} z=z \text { or } z^{d^{n}-1}+\cdots+\left(\lambda^{n}-1\right)=0
$$

if we ignore the fixed point at the origin. If $\mu_{1}, \ldots, \mu_{d^{n}-1}$ are the $d^{n}-1$ roots of this equation, then

$$
\left|\mu_{1}\right| \cdots\left|\mu_{d^{n}-1}\right|=\left|\lambda^{n}-1\right| .
$$

Let $m=\min \left|\mu_{i}\right|$. We get

$$
m^{d^{n}-1} \leqslant\left|\lambda^{n}-1\right| \leqslant(1 / n)^{d^{n}-1}
$$

for infinitely many natural numbers $n$. Hence, there are infinitely many periodic points converging to the origin. Yet, if the origin is in the Fatou set, (SFE) has a solution by (3.8), and the map $p(z)$ must be an irrational rotation near the origin. Since irrational rotations do not have periodic points (except the center), we conclude that the origin is in the Julia set.

It is natural to ask if

$$
\left|\lambda^{n}-1\right| \leqslant(1 / n)^{d^{n}-1}
$$

is ever satisfied for infinitely many $n$ if $\alpha \notin \mathbf{Q}$. To construct such $\lambda$, we use continued fraction expansions

$$
\left|\lambda^{n}-1\right|=\left|e^{2 \pi i n \alpha}-1\right|=\left|e^{\pi i n \alpha}-e^{-\pi i n \alpha}\right|=2|\sin (\pi i n \alpha)| .
$$

For each $n$, let $m_{n}$ be the integer such that $\left|n \alpha-m_{n}\right| \leqslant 1 / 2$. Using the fact that $2 x \leqslant \sin (\pi x) \leqslant \pi x \leqslant 7 x / 2$ when $|x| \leqslant 1 / 2$, we get

$$
\left|\lambda^{n}-1\right|=2|\sin (\pi i n \alpha)|=2 \sin \left(\pi\left|n \alpha-m_{n}\right|\right)
$$

and

$$
4\left|n \alpha-m_{n}\right| \leqslant\left|\lambda^{n}-1\right| \leqslant 7\left|n \alpha-m_{n}\right| .
$$

To find a dense set of $\lambda$ which satisfies (3.22), we specify any initial segment $\left[a_{0}, \ldots, a_{k}\right]$, and then we continue the expansion using the fact that

$$
\left|q_{l} \alpha-p_{l}\right|<\frac{1}{q_{l+1}}=\frac{1}{a_{l+1} q_{l}+q_{l-1}}<\frac{1}{a_{l+1} q_{l}} .
$$

If we inductively choose $a_{l+1}>7 q_{l}^{\left(d^{q}\right)-2}$, then equation (3.22) is satisfied for $n=q_{k+1}, q_{k+2}, q_{k+3}, \ldots$.

The reader is encouraged to write out the decimal expansion of an $\alpha$ that satisfies this condition. This example also shows that the complement of the set $\Lambda \cup$ \{roots of unity\} contains a dense subset of the unit circle.

In fact, the set of $\lambda$ 's which satisfy (3.22) for infinitely many $n$ is a residual subset of the unit circle.

LEMMA. Let $f(x)$ be any positive function defined on the positive integers. Then the set $\{\alpha<|| \alpha-(p / q) \mid<f(q)$ for infinitely many $p$ and $q\}$ is a residual subset of $\mathbf{R}$. 
Proof. Let $U_{N}=\{\alpha|| \alpha-(p / q) \mid<f(q)$ for some $q \geqslant N\}$. Then $U_{N}$ is an open, dense subset of the unit circle because it is the union of open disks and because it contains all but finitely many rationals. Therefore, $\bigcap_{N=1}^{\infty} U_{N}$ is a residual subset of $\mathbf{R}$.

Using this lemma and the above approximations, the reader can easily verify that the set of $\lambda$ 's which can be used in (3.21) is indeed residual.

We cannot yet always determine if a fixed point is in either the Julia set or the Fatou set because the neutral case has not been completely resolved. The hypothesis to Siegel's Theorem has been broadened somewhat (see Rüssman [Ru]), but there still are cases between Cremer's examples ((3.21)) and the known generalizations of Siegel's Theorem. Zehnder has also proven an $n$-dimensional version of Siegel's Theorem. These results suggest the following important open questions.

(3.23) Problems. (1) Is membership in the Fatou set entirely determined by the eigenvalue of the fixed point?

(2) What is the dynamics in a neighborhood of a periodic orbit if its eigenvalue is not a root of unity and (SFE) does not have a solution?

4. The consequences of Montel's Theorem. The following theorem of Montel is fundamental to proving that the Julia set is the closure of the repelling periodic points. In this section we use it to prove that $J$ is a perfect set. Therefore, it is uncountable.

(4.1) THEOREM. Let $\mathscr{F}$ be a family of meromorphic functions defined on a domain $U$. Suppose there exist points $a, b, c$ in $\overline{\mathbf{C}}$ such that $\left[\bigcup_{f \in \mathscr{F}} f(U)\right] \cap$ $\{a, b, c\}=\varnothing$. Then $\mathscr{F}$ is a normal family on $U$.

It is interesting to see that a condition on the images of a family could guarantee normality. However, the result is not surprising if it is considered in light of the uniformization theorem $((1.8))$ and the Poincare metric on the domain $\overline{\mathbf{C}}-\{a, b, c\}$. See [T2].

In this section we use Montel's Theorem to derive a few basic properties of the Julia set.

(4.2) Corollary. Let $z \in J(R)$. If $U$ is a neighborhood of $z$, then the set $E_{U}=\overline{\mathbf{C}}-\cup_{n>0} R^{n}(U)$ contains at most two points. Such points are called exceptional points.

(4.3) EXAmple. Consider a polynomial $p(z)$. The point at $\infty$ is very special. It is a fixed point whose only inverse image is itself. Very few fixed points have this property. In fact, if a rational map $R$ fixes a point $z_{0}$ and $R^{-1}\left(z_{0}\right)=\left\{z_{0}\right\}$, then $R$ can be conjugated by a Möbius transformation which sends $\infty$ to $z_{0}$. The result is a polynomial because it does not have any poles in $\mathbf{C}$.

The point at infinity is always a superattractive fixed point for $p(z)$. Hence, the Julia set of a polynomial is contained in $\mathbf{C}$. Since $\left[U_{n \geqslant 0} p^{n}(\mathbf{C})\right]=\mathbf{C}$, we see that $\infty$ is indeed an exceptional point. 
(4.4) Definition. Let $z \in J$. We define $E_{z}=\cup E_{U}$, where the union is taken over all neighborhoods $U$ of the point $z$. From (4.2), it follows easily that $E_{U}$ is independent of $U$, provided that $U$ is sufficiently small and $E_{z}$ contains at most two points.

The reader should note that $E_{z}$ is often empty.

(4.5) THEOREM. Let $E_{z}$ be the set of exceptional points for some $z \in J$.

(A) If $E_{z}$ contains two points, then $R(z)$ is conjugate to the map $z \rightarrow z^{ \pm d}$, where $d=\operatorname{deg}(R)$ and the sign is + if $E_{z}$ contains fixed points and - if $E_{z}$ consists of a periodic orbit of period two.

(B) If $E_{z}$ contains exactly one point, then $R(z)$ is conjugate to a polynomial. In both cases, it follows that $E_{z}$ does not depend on the choice of $z \in J$. Moreover, all exceptional points are contained in the Fatou set.

Proof. First note that $E_{z}$ is backwards invariant by definition. That is, $R^{-1}\left(E_{z}\right)=E_{z}$. Since $R$ is surjective, it follows that $E_{z}$ either consists of one fixed point, two fixed points, or an orbit of period two.

To prove (B) we argue just as in Example (4.3). Let $M$ be a Möbius transformation which maps the point in $E_{z}$ to the point at infinity. Then $p(z)=M \circ R \circ M^{-1}(z)$ has no poles in $\mathbf{C}$.

To prove (A) we use a Möbius transformation which moves one point of $E_{z}$ to $\infty$ and the other point to 0 . In this case the conjugated map $p(z)$ can be one of two forms. Suppose both 0 and $\infty$ are fixed by $p(z)$. Then (as above) $p(z)$ will be a polynomial. Moreover, 0 will be a zero of multiplicity $d$ because no other points can map to 0 . Hence, $p(z)=K z^{d}$, and the constant $K$ can be eliminated by conjugation with an expansion or a contraction. If 0 and $\infty$ form an orbit whose period is two, similar arguments show that $p(z)=K z^{-d}$.

To prove that $E_{z} \cap J=\varnothing$, we observe that, in case (A), the set $E_{z}$ consists of either two superattractive fixed points or a superattractive orbit of period two. In case (B), $E_{z}$ is a superattractive fixed point.

If $E_{z} \neq \varnothing$ for any $z$, we know that $R$ is conjugate to either (A) the map $z \rightarrow z^{ \pm d}$ or (B) a polynomial. If in case (A) we let $U=\overline{\mathbf{C}}-\{0, \infty\}$, and in case (B) we let $U=\mathrm{C}$, then $E_{U}=E_{z}$ for any $z \in J$. Consequently, $E_{z}$ is independent of $z$.

Notation. This theorem lets us simplify notation. The set of exceptional points will be denoted $E(R)$ or simply $E$ if the map is understood.

An easy consequence of (4.5) is the fact that $J$ does not usually have interior.

(4.6) Corollary. If $\operatorname{int}(J) \neq \varnothing$, then $J=\overline{\mathbf{C}}$.

Proof. Let $U$ be a domain contained in the interior of $J$. Since $J$ is forward invariant,

$$
J \supset \bigcup_{n \geqslant 0} R^{n}(U)=\overline{\mathbf{C}}-E
$$

Moreover, since $J$ is closed and $E$ contains at most two points, $J=\overline{\mathbf{C}}$. 
Another corollary is an inexpensive way to generate computer pictures of the Julia set.

(4.7) Corollary. If $z \in \overline{\mathbf{C}}-E$, then the Julia set is contained in the set of accumulation points of the full backwards orbit of $z$. That is,

$$
J \subset\left\{\text { accumulation points of } \bigcup_{n \geqslant 0} R^{-n}(z)\right\} .
$$

Consequently, if $z \in J$, then

$$
J=\operatorname{closure}\left(\bigcup_{n \geqslant 0} R^{-n}(z)\right) .
$$

Proof. The first statement follows immediately from the definition of $E$ and the fact that the iterates of any neighborhood of any point $w \in J$ must eventually hit $z$.

If $z \in J$, then $\bigcup_{n \geqslant 0} R^{-n}(z) \subset J$ because $J$ is backwards invariant. Moreover, since $J$ is closed,

$$
J \supset \operatorname{closure}\left(\bigcup_{n \geqslant 0} R^{-n}(z)\right) .
$$

The opposite inclusion follows from the first conclusion.

Almost all of the pictures in the preceding sections were generated by finding a repelling fixed point and then calculating its inverse orbit. This gives a dense subset of $J$. However, dense subsets can be deceiving, and consequently the more expensive methods of Mandelbrot and others often give better pictures.

PROBLEM. Design good algorithms to generate pictures of Julia sets.

In all the pictures we have seen, the Julia set appears to be infinite. We are now able to verify that it is uncountable.

(4.8) THEOREM. The Julia set is a perfect set.

Before proving the theorem, we establish a helpful lemma.

(4.9) Lemma. If $a \in J$, then there exists $b \in J$ such that $a \in O^{+}(b)$ but $b \notin O^{+}(a)$.

Proof OF LEMMA. If $a$ is not periodic, then $b$ can be any inverse image of $a$. If $a$ is periodic with period $n$, consider the map $S=R^{n}$ and the equation

$$
S(z)=a
$$

If $a$ is the only solution to (4.10), we may conjugate $S$ to a polynomial as in Example (4.3). Since $\infty$ is in the Fatou set of a polynomial, we have $a \in F(S)$, contradicting our assumption. Consequently, another solution $b$ to (4.10) exists, and $b \notin O^{+}(a)$ because $a$ is the only solution to (4.10) in $O^{+}(a)$. 
Proof of Theorem (4.8). Given $a \in J$, we prove that $a$ is an accumulation point of $J$. Let $U$ be a neighborhood of $a$. Choose $b$ as in Lemma (4.9). Since $b \in J, b \notin E$ and there exists an integer $k$ such that $b \in R^{k}(U)$. Let $c$ denote a point in $U$ such that $R^{k}(c)=b$. Then $c \neq a$ because $b \notin O^{+}(a)$, and $c \in J$ because $J$ is $R^{-1}$ invariant.

5. The Julia set is the closure of the set of repelling periodic points. Every repelling periodic point is contained in the Julia set, and since the Julia set is closed, the closure of the set of repelling periodic points is a subset of the Julia set. In this section we show that these two sets are equal. The proof has two parts. First, we show that $J$ is a subset of the closure of all periodic points. Then we derive a finite bound on the number of nonrepelling periodic orbits.

(5.1) Definition. The value $v$ is a critical value of $R$ if the equation $R(z)=v$ has a solution whose multiplicity is greater than one. Such a solution $c$ is called a critical point. The set $C$ will denote the set of all critical points of $R$, and $V$ will denote the set of critical values. Note that $R(C)=V$.

(5.2) REMARK. Using local coordinates, this is equivalent to the condition $R^{\prime}(c)=0$ (at least when $c \neq \infty$ ). Topologically we can characterize the critical points as the points where the map $R: \overline{\mathbf{C}} \rightarrow \overline{\mathbf{C}}$ is not locally injective. In other words, they are branch points of the branched cover $R: \overline{\mathbf{C}} \rightarrow \overline{\mathbf{C}}$. Recall that $d$ is the degree of $R$.

(5.3) Definitions. Given $z \in \overline{\mathbf{C}}$, the deficiency $d_{z}$ of $z$ is the number $d$ - (the cardinality of $R^{-1}(z)$ ). Then $d_{z} \neq 0$ if and only if $z$ is a critical value of $R$. The total deficiency of the map $R: \overline{\mathbf{C}} \rightarrow \overline{\mathbf{C}}$ is the sum of all the deficiencies for all values of $R$.

(5.4) LEMMA. The number of critical points of $R$ is at most $2 d-2$.

In the following proof, we actually prove that the total deficiency of $R$ is always $2 d-2$. Therefore, $d_{z}$ is usually 0 .

Proof. Let $S$ be $\overline{\mathbf{C}}-V$ and $\tilde{S}$ be $\overline{\mathbf{C}}-R^{-1}(V)$. The map $R: \tilde{S} \rightarrow S$ is a $d$-fold covering space. Therefore, we have the following relationship between their Euler characteristics:

$$
\chi(\tilde{S})=d \chi(S)
$$

This yields

$$
2-\left(\sum_{v \in V}\left(d-d_{v}\right)\right)=d\left(2-n_{v}\right)
$$

where $n_{v}$ equals the number of critical values. We get

$$
d n_{v}-\left(\sum\left(d-d_{v}\right)\right)=2 d-2
$$

$$
\sum_{v \in V} d_{v}=2 d-2
$$


Since each critical point causes at least one deficiency, we conclude that the cardinality of $C$ is at most $2 d-2$.

REMARK. The equation $2-\left(\sum_{v \in V}\left(d-d_{v}\right)\right)=d\left(2-n_{v}\right)$ is often called the Riemann-Hurewicz formula.

Now we can prove that the Julia set is contained in the closure of the periodic points.

(5.5) THEOREM. $J \subset$ closure $\{$ periodic points $\}$.

We give one proof that works for polynomials and then we show how to modify it to include rational functions. by

Proof. Let $p: \overline{\mathbf{C}} \rightarrow \overline{\mathbf{C}}$ be a polynomial. Consider the subset $K$ of $J$ defined

$$
K=J-\{\text { critical values of } p\}
$$

Since $J$ is perfect and $K$ differs from $J$ by only a finite subset, we can prove the theorem by showing that $K \subset$ closure\{periodic points\}.

Let $w \in K$. There exists a neighborhood $U$ of $w$ such that the polynomial $p$ has at least one local inverse $I: U \rightarrow \mathrm{C}-U$. Form the family of meromorphic functions $\left\{g_{n}\right\}$ by

$$
g_{n}(z)=\left(p^{n}(z)-z\right) /(I(z)-z) .
$$

Note that $\left\{g_{n}\right\}$ is a normal family if and only if the family $\left\{p^{n}\right\}$ is normal. Since $w \in J$, the family $\left\{g_{n}\right\}$ cannot be normal on any open subset $V$ of $U$. However, if $w$ were not in the closure of the set of periodic points, then $\left\{g_{n}\right\}$ would omit 0,1 , and $\infty$ on some neighborhood of $w$. This contradicts Montel's Theorem ((4.1)).

To generalize this proof to the case of rational functions we must use a slightly different family $g_{n}(z)$ and a slightly smaller set $K$. In that case we define

$$
K=J-\left\{\infty \text {, critical values of } R^{2} \text {, poles of } R^{2}\right\} .
$$

Then, given $w \in K$, we define

$$
g_{n}(z)=\frac{\left(R^{n}-I_{1}\right)}{\left(R^{n}-I_{2}\right)} \frac{\left(I_{3}-I_{2}\right)}{\left(I_{3}-I_{1}\right)},
$$

where $I_{1}, I_{2}$, and $I_{3}$ are three possible inverses to $R^{2}$ in a neighborhood $U$ of $w$. Since

$$
R^{n}=I_{2}+Q\left(\left(I_{2}-I_{1}\right) /\left(g_{n}-Q\right)\right)
$$

where

$$
Q=\left(I_{3}-I_{2}\right) /\left(I_{3}-I_{1}\right),
$$

we see that $\left\{g_{n}\right\}$ is a normal family if and only if $\left\{R^{n}\right\}$ is a normal family.

To finish proving that the Julia set is the closure of the set of repelling periodic points, we will use the theorem just proved along with the fact that the number of nonrepelling periodic points is finite. Since the Julia set is perfect 
((4.8)), every point in it will be the accumulation point of some sequence of repelling periodic points.

(5.6) Definition. Let $p$ be an attractive fixed point of $R$. Then the stable set (attractive basin) of $p$ is the set

$$
W^{s}(p)=\left\{z \mid R^{n}(z) \rightarrow p \text { as } n \rightarrow \infty\right\} .
$$

The immediate stable set $A(p)$ of $p$ is the maximal domain containing $p$ on which the family $\left\{R^{n}\right\}$ is normal.

To prove the next proposition, the reader should note that there are no proper, closed, completely invariant subsets of $J$.

(5.7) Proposition. The set $A(p)$ is the component of $W^{s}(p)$ containing $p$. Moreover, the frontier of $A(p)$ is contained in $J$ and the frontier of $W^{s}(p)$ is $J$.

(5.8) THEOREM. The immediate attractive set $A(p)$ contains at least one critical value.

To prove (5.8) we use the following lemma, which is an easy consequence of the monodromy theorem.

(5.9) Lemma. Suppose $S$ is a rational function and $D$ is a simply connected domain which does not contain any of the critical values of $S$. Given any $d \in D$ and any $c \in S^{-1}(d)$, there exists a unique analytic inverse $I$ to $S$ defined on $D$ such that $I(d)=c$.

Proof of Theorem (5.8). Suppose $A(p)$ does not contain any critical value. Take a simply connected open neighborhood $U$ of $p$ contained in $A(p)$. Apply the lemma to $R \mid U$ to get an inverse map $S_{1}$ such that $S_{1}(p)=p$. Then $S_{1}(U)$ is a subset of $A(p)$, and it is simply connected (note that $R$ and $S_{1}$ are analytic inverse homeomorphisms between $U$ and $S_{1}(U)$ ). Therefore, this process can be repeated infinitely often to get a family of functions $\left\{S_{k}\right\}$ on the set $U$. The family is a normal family by Montel's Theorem because $S_{k}(U) \subset A(p)$ for all $k$. This contradicts the fact that $p$ is a repelling fixed point for the maps $S_{k}$.

Of course, we may conclude that the number of attracting fixed points is at most $2 d-2$. Actually, using this reasoning, we can prove

(5.10) COROLLARY. The number of attracting periodic orbits is at most $2 d-2$.

To prove this, one applies the same argument as above with the following

(5.11) Definition. Let $p$ be an attracting periodic point of period $n$. Then the immediate attractive set $A(p)$ is the set $\bigcup_{k=0}^{n-1} A\left(R^{k}(p), R^{n}\right)$, where $A(x, S)$ represents the immediate attractive set of the attractive fixed point $x$ of the map $S$.

How do the neutral points relate to the attractive points? We get a bound on the number of neutral orbits by considering the following one-parameter family of rational functions. Let

$$
R(z, w)=(1-w) R(z)+w .
$$


(5.12) TheOREM. Suppose $R$ has $N$ neutral periodic points. There exist an $\varepsilon>0$ and a direction $\theta$ in the $w$ plane such that, if $0<\rho<\varepsilon$, the rational map

$$
R_{\rho}(z)=R\left(z, \rho e^{2 \pi i \theta}\right)
$$

has at least $N / 2$ attracting periodic points which are continuations of neutral orbits of $R$.

Proof. For each periodic point $z_{i}$ we let $n_{i}$ denote its period and $s_{i}$ its eigenvalue. The pair $\left(z_{i}, 0\right)$ satisfies the equation

$$
F_{i}(z, w)=R^{n_{i}}(z, w)-z=0 .
$$

Since $F_{i}(z, w)$ is a rational function of $z$ and $w$, there is an algebraic equation

$$
A_{i}(z, w)=p_{0}(w) z^{n}+p_{1}(w) z^{n-1}+\cdots+p_{n}(w)=0
$$

(where the $p_{i}(w)$ are polynomials) which is equivalent to $F_{i}(z, w)=0$. Applying the theory of algebraic functions to each equation $A_{i}(z, w)=0$, we can introduce new variables $w_{i}$ and analytic functions $z_{i}\left(w_{i}\right)$ such that

$$
w=w_{i}^{m_{i}}, \quad z=z_{i}\left(w_{i}\right) \quad \text { where } m_{i} \text { is a positive integer, }
$$

and

$$
A_{i}\left(z_{i}\left(w_{i}\right), w_{i}^{m_{i}}\right)=F\left(z_{i}\left(w_{i}\right), w_{i}^{m_{i}}\right)=0
$$

(see [N, pp. 47-55] for more details). Note that, when $s_{i} \neq 1$, this is the usual implicit function theorem. We can define derivative functions by

$$
s_{i}\left(w_{i}\right)=\frac{\partial R^{n_{i}}}{\partial z}\left(z_{i}\left(w_{i}, w_{i}^{m_{i}}\right)\right)
$$

Let $m$ be the least common multiple of all the $m_{i}$ corresponding to the $N$ neutral orbits. We introduce a new variable $v$ such that $v^{m}=w$. Then $v^{\left(m / m_{i}\right)}$ $=w_{i}$. The angle $\theta$ is obtained by calculating an angle $\theta_{1}$ in the $v$ plane.

To find $\theta_{1}$ write

$$
s_{i}(v)=s_{i}+a_{i} v^{k_{i}}+\cdots \quad \text { where } a_{i} \neq 0 .
$$

A nonzero higher order term exists because the map $z \mapsto 1$ does not have any neutral periodic orbits. Let

$$
\tilde{s}_{i}(v)=s_{i}+a_{i} v^{k_{i}}
$$

(5.13) Claim. If we find $\varepsilon_{1}>0$ and $\theta_{1}$ such that at least half of the values satisfy $\left|\tilde{s}_{i}\left(\rho e^{2 \pi i \theta_{1}}\right)\right|<1$, then we can use $\theta_{1}$ as our appropriate direction, which will yield the same result for $s_{i}$.

We shall verify the hypothesis of the Claim and leave the rest to the reader.

Let $2^{l}$ be the highest power of two that divides any of the $m_{i}$. For each $s_{i}$ on the unit circle, consider the tangent direction and the directions which differ from the tangent by an angle which is a multiple of $\pi / 2^{l}$. Figure (5.13a) illustrates the case where $l=1$ and there are three neutral orbits.

By considering all such $s_{i}$, we obtain a finite set of directions (at most $N$ times $2^{l+2}$; see Figure (5.13b)). 


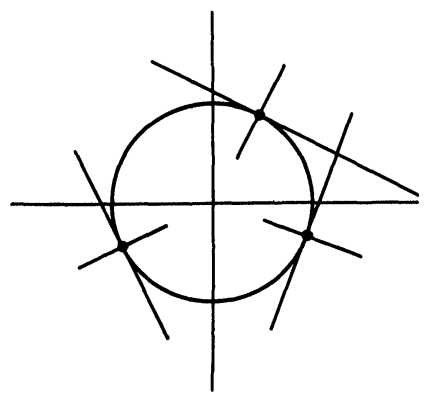

(5.13a) FigURE

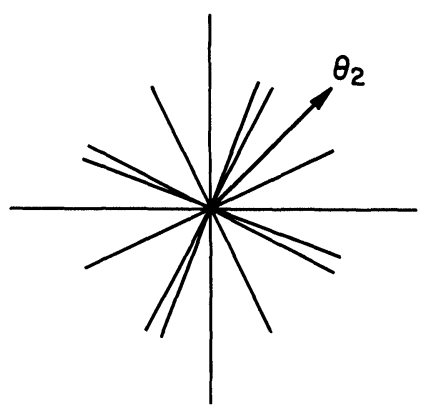

(5.13b) FigURE

Choose any angle $\theta_{2}$ which is not one of these directions. The angle $\theta_{1}$ that we seek will be $\theta_{1}=\theta_{2}+\theta_{3}$, where $\theta_{3}$ is determined in an inductive fashion. The angle

$$
\theta_{3}=\pi\left(b_{0}+b_{1} / 2+\cdots+b_{l} / 2^{l}\right)
$$

where $b_{i} \in\{0,1\}$. We determine $b_{i-1}$ after we have calculated $b_{i}, \ldots, b_{l}$.

Consider all periodic points $z_{i}$ whose periods $n_{i}$ are divisible by $2^{i}$. Then

$$
\tilde{s}_{i}\left(\rho e^{2 \pi i \theta_{1}}\right)=s_{i}+\rho^{k_{i}} e^{2 \pi i\left(k_{i} \theta_{2}+\pi b_{l}\right)} .
$$

Since $k_{i} \theta_{2}$ is a direction which is not tangent to the unit circle, we choose $b_{l}$ such that at least half of the $\tilde{s}_{i}(v)$ go inside the unit circle.

To determine $b_{l-1}$, repeat this calculation on all orbits $z_{i}$ for which $k_{i}$ is divisible by $2^{l-1}$ but not by $2^{l}$. Then

$$
\tilde{s}_{i}\left(\rho e^{2 \pi i\left(\theta_{2}+\theta_{3}\right)}\right)=s_{i}+\rho^{k_{i}} e^{2 \pi i\left(k_{i} \theta_{i}+\pi b_{l-1}+(q / 2) \pi b_{l}\right)}
$$

where $q$ is some odd number.

Since $k_{i} \theta_{1}+(q / 2) \pi b_{l}$ is not tangent to the unit circle, we choose $b_{l-1}$ so that at least half of these orbits go inside the circle. We just continue this induction until the angle $\theta_{1}$ is determined.

When a rational map with (super)attractive periodic orbits is varied along a one-parameter family, these orbits will remain for, at least, some small interval. Therefore, we can combine (5.10) with (5.12) to get the classical bound stated below. 
(5.14) COROLLARY. The number of attracting periodic orbits plus half the number of neutral periodic orbits is at most $2 d-2$.

These estimates are not the whole story. Douady and Hubbard (using their theory of polynomial-like functions) have improved them in many cases (see (11.12)). But (5.14), when combined with (5.5), is enough to prove the following fundamental decomposition theorem.

(5.15) THEOREM. The Julia set equals the closure of the repelling periodic points.

One immediate consequence of (5.15) is the property that neighborhoods of points in $J$ are eventually surjective (i.e. $J$ is "locally, eventually onto" (leo)).

(5.16) Corollary. Let $A$ be a closed subset of $\overline{\mathbf{C}}$ such that $A \cap E=\varnothing$. Given a neighborhood $U$ of a point $p \in J$, there exists an integer $N$ such that $A \subset R^{N}(U)$. Therefore, if $D$ is a domain such that $D \cap J \neq \varnothing$, then there exists an $N$ such that $R^{N}(D \cap J)=J$.

Proof. Let $q \in U$ be a repelling periodic point of period $n$. Choose a neighborhood $V$ of $q$ such that $V \subset U$ and $R^{n}(V) \supset V$. Since $A \cap E=\varnothing$ and $q \in J$,

$$
A \subset \bigcup_{k=1}^{\infty} R^{k n}(V)
$$

By construction,

$$
R^{n}(V) \subset R^{2 n}(V) \subset R^{3 n}(V) \subset \cdots .
$$

Since $A$ is compact, $A \subset R^{N}(V) \subset R^{N}(U)$ for some $N=k n$.

In the classical papers this result is often referred to as local homogeneity. Sullivan (see (8.6)) has strengthened this result when $R \mid J$ is expanding.

6. Classical results concerning the Fatou set. The papers of Fatou and Julia contain many results beyond the fundamental decomposition theorem. In this section we mention two of their theorems regarding the components of the Fatou set.

Recall that a set $D$ is completely invariant if both $R(D)=D$ and $R^{-1}(D)=D$.

(6.1) Proposition. Let $D$ be a simply connected, completely invariant component of the Fatou set. Then the total deficiency (see (5.3)) of the map $R: D \rightarrow D$ is $d-1$.

Proof. This is proven using the same covering space theory as in the proof of Lemma (5.4). Viewing $R: D \rightarrow D$ as a $d$-fold branched cover, we get

$$
1-\left(\sum_{v \in V \cap D}\left(d-d_{v}\right)\right)=d\left(1-n_{v}\right)
$$


and conclude that

$$
\left(\sum_{v \in V \cap D} d_{v}\right)=d-1
$$

(6.2) Corollary. The Fatou set cannot contain more than two different, completely invariant, simply connected components.

With this observation we can count the number of components in the Fatou set.

(6.3) Lemma. The frontier of any completely invariant component of the Fatou set is the Julia set.

Proof. The frontier is a nonempty, closed, completely invariant subset of $J$. Therefore, it must be all of $J$.

(6.4) Definitions. Let $D_{i}$ be a component of the Fatou set. If there exists an integer $n_{i}$ such that $R^{n_{i}}\left(D_{i}\right)=D_{i}$, then $D_{i}$ is periodic. Another component $D_{j}$ is eventually periodic if there exists $n_{j}$ such that $R^{n_{j}}\left(D_{j}\right)$ is periodic. The domain $D_{j}$ is preperiodic if it is eventually periodic but not periodic.

(6.5) REMARK. Since $R$ must be surjective, the existence of one preperiodic component in the Fatou set implies the existence of infinitely many preperiodic components.

(6.6) THEOREM. If the number of components of $F$ is finite, then there are at most two.

Proof. Assume that the number of components is finite but greater than two. Using (6.5) we obtain an integer $n$ such that the rational map $S_{1}=R^{n}$ setwise fixes each component of $F$. Therefore, each component is completely invariant, and one (denoted $A$ ) is not simply connected. Choose a point $z$ in another component $D$ and a Möbius transformation $M$ moving $z$ to $\infty$. Then conjugate $S_{1}$ by $M$ to get the rational map $S_{2}$ which has $\infty$ in a completely invariant component of $F$. The Fatou set of $S_{2}$ also contains a multiply connected, completely invariant component $A^{\prime}$ in $\mathbf{C}$.

Take a loop $l$ in $A^{\prime}$ which does not bound a disk in $A^{\prime}$. Since $l \subset A^{\prime}$ and $A^{\prime}$ is invariant, the iterates $S_{2}^{k}$ are uniformly bounded on $l$. Since these iterates are analytic on the finite component $K$ of $\overline{\mathbf{C}}-l$, we can use the Cauchy integral formula to conclude that the family $S_{2}^{k}$ is uniformly bounded on $K$. Using (1.6) we see that this contradicts the fact that $K \cap J \neq \varnothing$.

In $\$ 2$ we saw examples of all possibilities except $F=\varnothing$. Figure (2.3) illustrates a Fatou set which is equal to $A(\infty)$. The Julia set in Figure (2.2) divides $\overline{\mathbf{C}}$ into two simply connected, completely invariant domains, and the Fatou set of Douady's Rabbit (Figure (2.1)) contains preperiodic domains.

In $\S 3$ the Weierstrass $\wp$ function was used to construct an example where $F=\varnothing$, and in the next section Sullivan's classification theorem will be used to find more examples where $F=\varnothing$ (see (7.9)).

The immediate attractive set $A(\infty)$ in Figure (2.3) is of infinite connectivity, and $A(0)$ in Figure (2.2) is simply connected. This is all that can happen. 


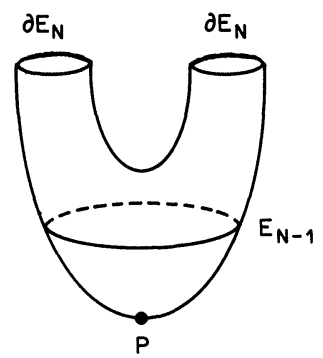

(6.8) Figure. A typical $E_{N}$.

(6.7) ThEOREM. Let $p$ be an attractive fixed point. Then $A(p)$ is either simply connected or of infinite connectivity.

Proof. Choose a simply connected, closed disk $D \subset A(p)$ such that $p \in D$, $R(D) \subset \operatorname{int}(D)$, and

$$
(\partial D) \cap\left(\bigcup_{\substack{\text { all critical } \\ \text { points } c}} O^{+}(c)\right)=\varnothing .
$$

Inductively define $E_{i}$ by $E_{0}=D$ and $E_{n}=R^{-1}\left(E_{n-1}\right) \cap A(p)$. Then

$$
E_{0} \subset E_{1} \subset \cdots \text { and } A(p)=\bigcup_{n=0}^{\infty} E_{n} \text {. }
$$

If $A(p)$ is not simply connected, there exists an integer $N$ such that $E_{N}$ is not simply connected. Choose $N$ to be the first integer such that $E_{N}$ is not simply connected. Then $E_{N}$ is an orientable, two-dimensional manifold with boundary, and $\partial E_{N}$ has at least two components. See Figure (6.8).

The boundary of $E_{N}$ separates $\overline{\mathbf{C}}-E_{N}$ into at least two components. Repeatedly consider the branched covers $R: E_{N+k+1} \rightarrow E_{N+k}(k=0,1,2, \ldots)$. They are actually covering spaces if $\left(E_{N+k+1}-E_{N+k}\right)$ does not contain any critical points, but nonetheless the number of boundary curves of $E_{N+k}$ is at least $2^{k+1}$. See Figure (6.9).

Consequently, $\partial A(p)$ will have infinitely many components and $A(p)$ is of infinite connectivity.

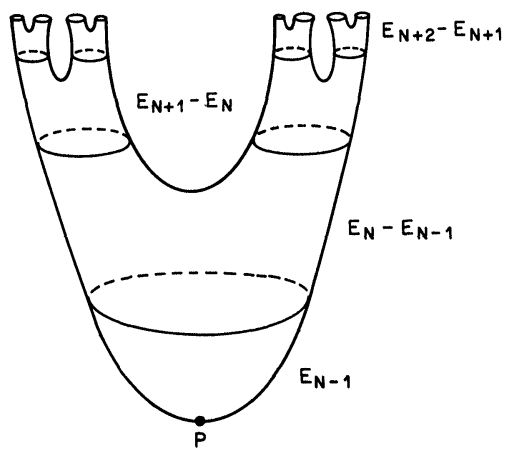

(6.9) FIGURE. A typical $E_{N+2}$. 
7. Sullivan's classification of the Fatou set. Recently Sullivan has completed a classification of the dynamical possibilities of $R \mid F[\mathbf{S 1}, \mathbf{S 2}]$. His theorems combine results of Fatou, Julia, Siegel [Si, SM], Arnold [Ar], Moser, and Herman on complex analytic dynamics with the theory of quasi-conformal homeomorphisms (see Ahlfors [A3] and Lehto and Virtanen [LV]) to yield a simple picture of $R \mid F$. The classification can be summarized in two theorems ((7.1) and (7.7)) which we will not prove (see (6.4) for relevant definitions). A discussion of quasi-conformality and dynamics is contained in $\$ 11$.

\section{(7.1) THEOREM. Every component of the Fatou set is eventually periodic.}

The Fatou set often contains preperiodic components in addition to the periodic ones (see (6.5) and (6.6)). However, we usually focus our attention on the periodic components. To make this distinction easier to discuss, we introduce the following terminology.

(7.2) Definition. A Sullivan domain of $R: \overline{\mathbf{C}} \rightarrow \overline{\mathbf{C}}$ is a periodic component of the Fatou set.

So we need only consider the dynamics in the Sullivan domains in order to understand the dynamics of $R \mid F$.

Sullivan's second theorem classifies the dynamics of $R$ restricted to a Sullivan domain. Five kinds of dynamics are possible.

(7.3) Definitions. Let $D$ be a Sullivan domain of period $n$ and let $S=R^{n}$.

(1) $D$ is an attracting domain if $D$ contains a periodic point $p$ such that $0<\left|S^{\prime}(p)\right|<1$ and $D=A(p, S)$.

(2) $D$ is a superattracting domain if $D$ contains a periodic point $p$ such that $p$ is a critical point of $S$ (i.e. $\left.S^{\prime}(p)=0\right)$ and $D=A(p, S)$.

(3) $D$ is a parabolic domain if there exists a periodic point $p$ in $\partial D$ whose period divides $n$ and $S^{k}(z) \rightarrow p$ as $k \rightarrow \infty$ for all $z \in D$.

(4) $D$ is a Siegel disk if $D$ is simply connected and $S \mid D$ is analytically conjugate to a rotation. In other words, there exists an analytic homeomorphism $h: D \rightarrow D_{r}$ such that the diagram

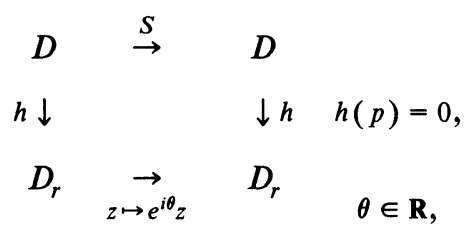

commutes.

(5) $D$ is a Herman ring if $D$ is conformally equivalent to an annulus $A=\left\{z \in \mathbf{C}\left|r_{1}<\right| z \mid<r_{2}\right\}$ (where $r_{1}, r_{2} \in \mathbf{R}, r_{1} \geqslant 0, r_{2}>0$ ) and the map $S \mid D$ is analytically conjugate to a rigid rotation of the annulus.

Siegel disks and Herman rings are often referred to as rotation domains.

(7.4) Remarks. (1) In the definitions of Siegel disk and Herman ring, it is enough to specify that the maps be topologically conjugate to rotations. Topological conjugacy implies analytic conjugacy. 
(2) Since $\operatorname{deg}(R) \geqslant 2$ and $R$ is analytic, $S \mid D$ can never be conjugate to a rotation $z \mapsto e^{i \theta} z$ where $\theta$ is rational (see (3.9)).

The Sullivan domains in Figure (2.1) are superattracting, and the finite Sullivan domain in Figure (2.2) is a typical attracting domain. Parabolic domains are intimately connected with the Flower Theorem ((3.12)) (see Figures (3.13)-(3.16)). Because the rotation domains exist in maps which satisfy delicate number theory conditions, we have not made any computer pictures of them. However, using Siegel's Theorem ((3.19)), it is easy to exhibit polynomial examples which have Siegel disks as Sullivan domains. Let $p(z)=$ $e^{2 \pi i \alpha} z+\cdots+z^{d}$, where $\alpha$ satisfies the number-theoretic condition in (3.19). Then the origin is a neutral fixed point which is the center of a Siegel disk.

Finding examples of rational maps which have Herman rings as Sullivan domains is more difficult. Using Arnold's theorems [Ar] on the existence of analytic conjugacies to irrational rotations, it is possible to find values of $a$ and $\alpha$ such that the map

$$
z \mapsto \frac{e^{2 \pi i \alpha}}{z}\left(\frac{z-a}{1-\bar{a} z}\right)^{2}
$$

has a Herman ring which contains the unit circle. For more examples, see Herman [H1].

Figures (7.5)-(7.6) give some idea of how these rotation domains should look.

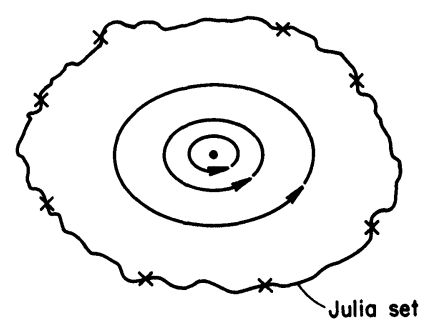

(7.5) Figure. A Siegel disk. The disk has a "foliation" by invariant curves. The boundary of the disk is contained in the closure of the forward orbits of the critical points. The $x$ 's in the figure represent points in these orbits.

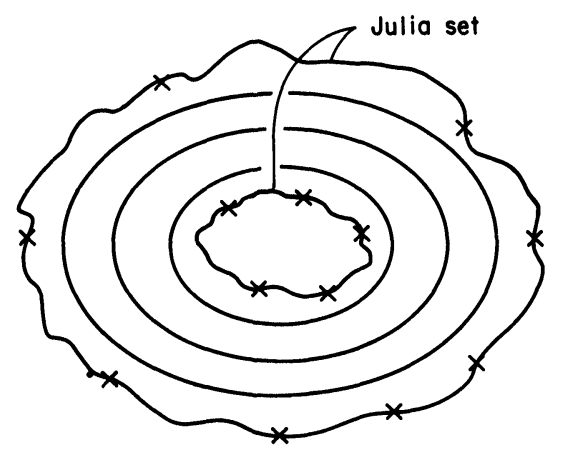

(7.6) FigURE. A Herman ring. There are many properties of Herman rings which are analogous to those of Siegel disks. The domain is foliated by invariant curves and the boundary of the ring is contained in the closure of the forward orbits of the critical points (again marked with $x$ 's). 
Sullivan's second theorem combines his work with the results referred to in the first paragraph of this section to finish the classification of the Fatou set.

(7.7) THEOREM. Every Sullivan domain is either attracting, superattracting, parabolic, a Siegel disk, or a Herman ring. Furthermore, there are finitely many such domains. In the parabolic case, $S^{\prime}(p)=1$. The attracting and parabolic domains both contain infinite forward orbits of critical points, and the boundaries of rotation domains are contained in the closure of the forward orbits of the critical points.

One very important problem concerning the number of Sullivan domains remains.

(7.8) Problem. Given a rational map of degree $d$, can it have more than $2 d-2$ orbits of Sullivan domains?

The classification has many interesting consequences.

(7.9) COROllaRY. Suppose every critical point of $R$ is preperiodic. Then $J(R)=\overline{\mathbf{C}}$.

Proof. Since every Sullivan domain except the superattracting ones requires critical points with infinite orbits, and since superattracting domains contain periodic critical points, $F(R)$ does not contain any Sullivan domains.

EXAMPLE. The rational function $R(z)=(z-2)^{2} / z^{2}$ satisfies the hypothesis of (7.9).

REMARK. Mary Rees [Re2] has proven a measure-theoretic version of (7.9). She has shown that, if all the critical points of $R$ are preperiodic and are eventually mapped onto repelling periodic points, then $R$ is ergodic.

In the polynomial case the argument in (7.9) can be used to show that $F$ consists of exactly one simply connected component $A(\infty)$.

(7.10) COROLlaRY. Let $p(z)$ be a polynomial and suppose all critical points except $\infty$ are preperiodic. Then $\overline{\mathbf{C}}=J \cup A(\infty)$.

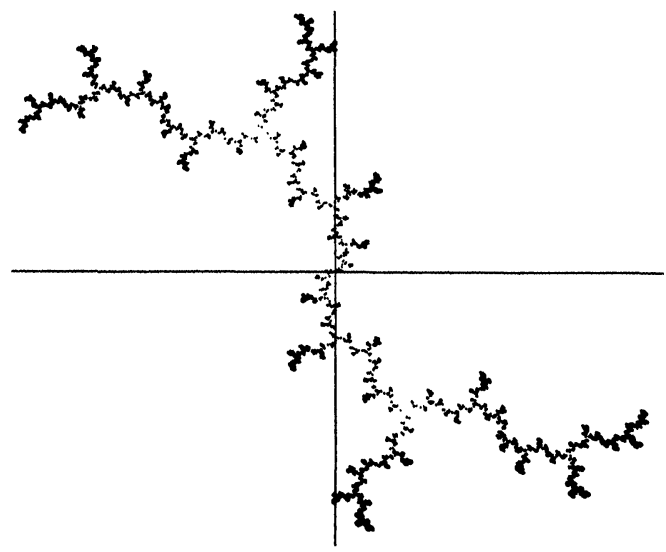

(7.13) FIGURE. The Julia set of $z^{2}+i$. 
Proof. The argument in (7.9) proves that $\overline{\mathbf{C}}=J \cup W^{s}(\infty)$. But since $W^{s}(\infty)=A(\infty)$ for polynomials, the result follows.

(7.11) Definition. Whenever $F$ consists of exactly one connected, simply connected set and $J$ is locally connected (for definition see (10.12)), we say that $J$ is a dendrite.

(7.12) ExAmples. (1) The polynomial $z \mapsto z^{2}-2$ satisfies the hypothesis of Corollary (7.10) and $J=\{z \mid z \in \mathbf{R}$ and $-2 \leqslant z \leqslant 2\}$.

(2) The Julia set of the polynomial $z \mapsto z^{2}+i$ is also a dendrite. See Figure (7.13).

(7.14) EXAMPLE. Let $p(z)=\lambda z+z^{2}$, where $\lambda=e^{2 \pi i \theta}$ and $\theta \notin \mathbf{Q}$ but is very nearly approximated by rationals (the exact diophantine condition is given in (3.21)). Then $F(p)$ also only has one component. However, $J(p)$ is not locally connected. To prove this, one needs results of Douady and Hubbard regarding polynomial-like maps as well as the classification of $F$. See (9.4) for more details.

8. A condition for expansion on the Julia set. Given the success of the last twenty years in the study of expanding and hyperbolic systems, it would be useful to know if $R \mid J$ is expanding. In our context there is a relevant classical result. We shall need it in the next section, and it is also related to the structural stability results of Mañé, Sad, and Sullivan [MSS].

(8.1) THEOREM. Let $\overline{O^{+}(C)}$ denote the closure of the forward orbits of the critical points. If $\overline{O^{+}(C)} \cap J=\varnothing$, then, given $K>1$, there exists an integer $N$ such that $\left|\left(R^{n}\right)^{\prime}(z)\right|>K$ if $n \geqslant N$ and $z \in J$.

To prove this, we consider sequences of inverse functions of the family $\left\{R^{n}\right\}$. Recall ((5.9)) that if $D$ is a simply connected domain which does not contain a critical value of $R$, then there exist at least two inverse functions to $R$ defined on $D$. Suppose, in addition, $D$ is disjoint from the forward orbits $O^{+}(C)$ of all critical points of $R$. Then all the iterates $R^{n}$ can be inverted on $D$. In the proof of the theorem, we use this observation and consider sequences $\left\{I_{j}: D \rightarrow \overline{\mathbf{C}}\right\}$ of inverse functions defined on simply connected domains $D$ which are disjoint from $O^{+}(C)$ and which satisfy $I_{0}=\operatorname{Id}$ and $R \circ I_{j}=I_{j-1}$.

(8.2) LEMMA. Let $D \cap \overline{O^{+}(C)}=\varnothing$ and let $\left\{I_{j}\right\}$ be a sequence of inverse functions as defined above. If $J \cap \overline{O^{+}(C)}=\varnothing$, then any convergent subsequence of $\left\{I_{j}\right\}$ converges to a constant function.

Proof OF (8.2). First we prove that the set $\left\{I_{j}\right\}$ is a normal family on $D$. Choose a repelling periodic orbit $O^{+}(p)$. Since $p$ is not an exceptional point, there are two distinct points $p_{1}$ and $p_{2}$ which are also distinct from $p$ such that $R\left(p_{1}\right)=p$ and $R\left(p_{2}\right)=p_{1}$. To prove that $\left\{I_{j}\right\}$ is a normal family on $D_{p}=D-O^{+}(p)$, note that $I_{j} \mid D_{p}$ must omit $\left\{p, p_{1}, p_{2}\right\}$ if $j>2$. That is, if $I_{j}(z)=p$, then $z=R^{j}\left(I_{j}(z)\right)=R^{j}(p) \in O^{+}(p)$. To prove normality on all of $D$, choose a different repelling periodic orbit $O^{+}(q)$ and prove normality on $D_{q}=D-O^{+}(q)$. Since $D=D_{p} \cup D_{q}$, the family $\left\{I_{j}\right\}$ must be normal on $D$. 
The assumption $J \cap \overline{O^{+}(C)}=\varnothing$ implies $F \neq \varnothing$ and the Sullivan domains are either attracting or superattracting domains. Consequently, the forward orbits of all points in $D \cap F$ accumulate on a finite set of periodic points which are contained in $\overline{O^{+}(C)}$.

Now, let $D_{-k}$ denote $I_{k}(D)$. We show that $D_{-k}$ converges uniformly to $J$ as $k \rightarrow \infty$. If not, there would exist two sequences $k_{i}$ and $z_{i}$ such that $R^{k_{i}}\left(z_{i}\right) \in D$, yet all of the $z_{i}$ are outside some neighborhood of $J$. Let $z_{*}$ be an accumulation point of the $z_{i}$. Then $\left\{R^{k}\right\}$ is a normal family on some neighborhood of $z_{*}$. Choose a convergent subsequence $\left\{R^{k_{i}}\right\}$ of the sequence $\left\{R^{k_{i}}\right\}$ and consider the images $R^{k_{i}}\left(z_{*}\right)$. Since the sequence converges uniformly, we can conclude that $R^{k_{i}}\left(z_{*}\right)$ converges to some point in $D$. But this contradicts the fact that

$$
D \cap\{\text { attracting and superattracting periodic orbits }\}=\varnothing .
$$

We have a normal family $\left\{I_{j}\right\}$ whose images $D_{-j}$ are converging uniformly to the Julia set. However, the Julia set does not contain interior. Therefore, any limit function has to be a constant function since its image does not contain interior.

Given this lemma, it is not difficult to prove the theorem.

Proof of Theorem (8.1). Cover the Julia set $J$ with a finite number of simply connected domains $D_{i}$ whose closures are disjoint from $\overline{O^{+}(C)}$. By the lemma, any sequence of inverse functions $I_{j}: D_{i} \rightarrow \overline{\mathrm{C}}$ must limit to a constant function. Therefore, the derivatives $I_{j}^{\prime}$ limit to zero. From this, the result follows because we can obtain an $N$ such that $1 /\left|\left(R^{n}\right)^{\prime}(z)\right|<1 / K$ for all $n \geqslant N$ and all $z \in D_{i}$.

The proof of the lemma is useful in the actual classification theorem. We stated that the boundary of a rotation domain $D$ is contained in the closure of the forward orbits of the critical points. We now briefly show why the proof of this fact is essentially the same as the argument in the proof of Lemma (8.2).

Proof OF PART OF TheOReM (7.7). Suppose $z_{0}$ is a point in $\partial D$ but not in the closure of the forward orbits of the critical points. Using the leo property ((5.16)) of the Julia set and the fact that the inverse images of rotation domains contain preperiodic domains, we can find a path $l$ from inside the rotation domain to a point $z_{1}$ in a preperiodic component of the Fatou set such that $l \cap \overline{O^{+}(C)}=\varnothing$. See Figure (8.3).

Using $l$ find a simply connected domain $D_{1}$ containing $l$ but disjoint from $\overline{O^{+}(C)}$. We define a sequence of inverse functions $I_{j}: D_{1} \rightarrow \overline{\mathbf{C}}$ such that $I_{j}\left(z_{2}\right) \in D$ for all $j$.

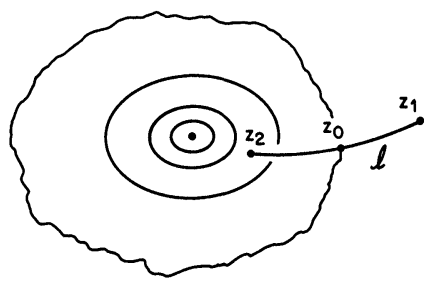

(8.3) FiguRE. The rotation domain $D$ and the path $l$. The point $z_{1}$ is in a preperiodic component of $F$; the point $z_{0}$ is in the boundary of $D$; and the point $z_{2}$ is an element of the rotation domain. 
A contradiction is derived using the limit functions of the $I_{j}$. Consider $I_{j}: D_{2} \rightarrow \overline{\mathbf{C}}$ and $I_{j}: D_{3} \rightarrow \overline{\mathbf{C}}$, where $D_{2}$ and $D_{3}$ are subsets of $D_{1}$, but $D_{2} \subset D$ and $D_{3} \cap D=\varnothing$. See Figure (8.4).

Consequently, the functions limit to constant functions. However, $I_{j}: D_{2} \rightarrow \overline{\mathbf{C}}$ is determined by an irrational rotation and therefore the limits are never constants. Since a constant function can never be analytically continued to a nonconstant one, we derive a contradiction.

In $\$ 5$ we referred to the leo property as a justification of the statement "Julia sets are fractals". This statement is not precisely true all the time, but Sullivan has a simple description of the self-similarity when $R \mid J$ is expanding.

(8.5) Definitions. A function $f: X \rightarrow X$ of a metric space $X$ with metric $d$ is a $K$-quasi-isometry if $(1 / K) d(x, y)<d(f(x), f(y))<K d(x, y)$ for all $x, y$ in $X$. The Julia set $J(R)$ is quasi-self-similar if there exists a $K$ and $r_{0}$ such that $\phi_{r}\left(J \cap D_{r}(x)\right)$ maps into $J$ by a $K$-quasi-isometry for all $r<r_{0}$ and all $x \in J$ (here $\phi_{r}$ is multiplication by $1 / r$ ).

In other words, a set is quasi-self-similar if there exists a $K$ such that every small piece can be expanded to full size and then placed onto $J$ by a $K$-quasi-isometry.

(8.6) Theorem (Sullivan [S3, S4]). If $J \cap \overline{O^{+}(C)}=\varnothing$, then $J$ is quasiself-similar.

9. The dynamics of polynomials. Some of the special characteristics of the dynamics of polynomials have already been described, but since they form a particularly important class of systems, they are worthy of more attention. In this section, we discuss results which apply to all polynomials regardless of their degree.

Let $p(z)$ be a polynomial of degree $d$. As we have frequently mentioned, the point at infinity plays a distinguished role. It is both an exceptional point and a superattractive fixed point whose deficiency (see (5.3)) is $d-1$. Consequently, $F(p) \neq \varnothing$ always; $J(p)$ is contained in a bounded subset of the complex plane; and the immediate stable set $A(\infty)$ equals the entire stable set $W^{s}(\infty)$. Moreover, there is a neighborhood $U$ of infinity and a real number $r \geqslant 1$ such that $p \mid U$ is analytically conjugate to the map $z \mapsto z^{d}$ restricted to the set $\overline{\mathbf{C}}-D_{r}$.

Since infinity is a critical value whose deficiency is $d-1$, the proof of Lemma (5.4) tells us there are at most $d-1$ finite critical points. The behavior

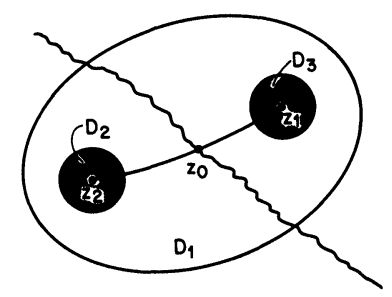

(8.4) Figure. The disks $D_{1}, D_{2}$, and $D_{3}$ in a subset of the region shown in (8.3). The proof and, therefore, the conclusion of Lemma (8.2) apply to $I_{j}: D_{3} \rightarrow \overline{\mathbf{C}}$. 
of these critical points often determines a great deal about the dynamics. As a result it is handy to distinguish between the set of all critical points $C$ and the set of finite critical points $C^{\prime}$. Because there are at most $d-1$ points in $C^{\prime}$, we can conclude (using (5.8) and (5.12)) that the number of finite attracting periodic orbits plus half of the number of neutral periodic orbits is at most $d-1$.

(9.1) Remark. Douady and Hubbard (see [D]), using the theory of polynomial-like functions, have sharpened this count of nonrepelling orbits. There are at most $d-1$ such orbits. See $\$ 11$.

The Fatou set of a polynomial is somewhat simpler than the general case.

\section{(9.2) THEOREM. The Sullivan domains of a polynomial are never Herman rings.}

Proof. We prove the theorem by applying the maximum principle to the family of functions $\left\{p^{n}\right\}$. Suppose one of the Sullivan domains $A$ was a Herman ring. Let $l$ denote any of the invariant Jordan curves in $A$. See Figure (9.3). Let $U$ be the open disk bounded by $l$ and not containing infinity. Since $U \cap J(p) \neq \varnothing$ and we are not dealing with the case where the cardinality of $E(p)$ is two, we conclude that $U p^{n}(U)=\mathbf{C}$. However, this conclusion contradicts the maximum principle applied to the functions $p^{n} \mid U$ because $p^{n}(l)=l$.

(9.4) Remark. Douady's improved bound of the number of nonrepelling periodic orbits $((9.1))$ is the missing ingredient necessary to prove (7.14). If $f_{\lambda}(z)=\lambda z+z^{2}$ and $|\lambda|=1$, but $\lambda$ is not a root of unity, then either $f_{\lambda}$ is linearizable in a neighborhood of 0 (i.e. (SFE) has a solution) or $F\left(f_{\lambda}\right)$ is connected. Like (7.10) we prove this by analyzing the possible Sullivan domains. Since 0 is nonrepelling, (9.1) implies there are no (super)attracting domains, except the one containing infinity, and there are no parabolic domains. The only available candidate for a finite Sullivan domain is a Siegel disk. Then $f_{\lambda}$ would be linearizable around 0 (see (3.20)).

In the beginning of this section we mentioned that there is a conjugacy between $p(z)$ and $z \mapsto z^{d}$ in some neighborhood of infinity. A great deal of information can be derived from attempting to extend this conjugacy over as large a region as possible. In fact, the rest of this section is essentially a treatment of this question.

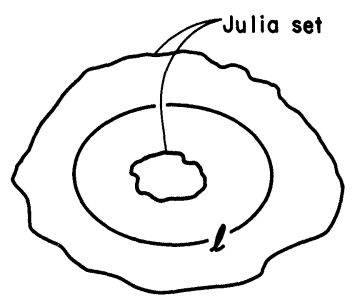

(9.3) Figure. The possible Herman ring. 
(9.5) THEOREM. The following statements are equivalent.

1. The map $p \mid A(\infty)$ is analytically conjugate to the map $z \mapsto z^{d}$ restricted to the exterior of the unit circle.

2. The set $A(\infty)$ is simply connected.

3. The Julia set $J(p)$ is connected.

4. The sets $A(\infty)$ and $C^{\prime}$ are disjoint. In other words, for every finite critical point $z$, the sequence of successive images $p^{n}(z)$ remains bounded.

Proof. $1 \Rightarrow 2$. The conjugacy is a homeomorphism between a disk and $A(\infty)$.

$2 \Rightarrow 3$. A plane set is simply connected if and only if its frontier is connected (see Newman [Ne]). The implication follows since $\partial A(\infty)=\partial W^{s}(\infty)=J(p)$.

$3 \Rightarrow 4$. We prove this implication by showing that $A(\infty) \cap C^{\prime} \neq \varnothing$ implies $J(p)$ is disconnected. Let $h: U \rightarrow D_{r}$ denote the conjugacy between $p(z)$ and $z \mapsto z^{d}$ in a neighborhood of $\infty$, and let $A_{0}$ be the annulus $h^{-1}\left(D_{r d}-D_{r}\right)$. Using the conjugacy, we construct an orthogonal coordinate system on $U$ where circles concentric to $\partial U$ are wrapped by $p(z)$ onto other such circles in a $d$-to-1 fashion. Radii are mapped to other radii. See Figure (9.6).

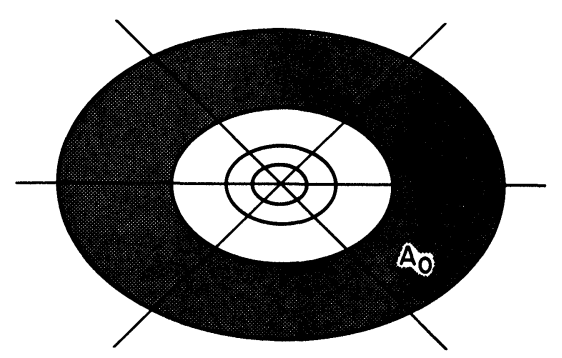

(9.6) FIGURE. The orthogonal coordinate system on $U$ and the annulus $A_{0}$.

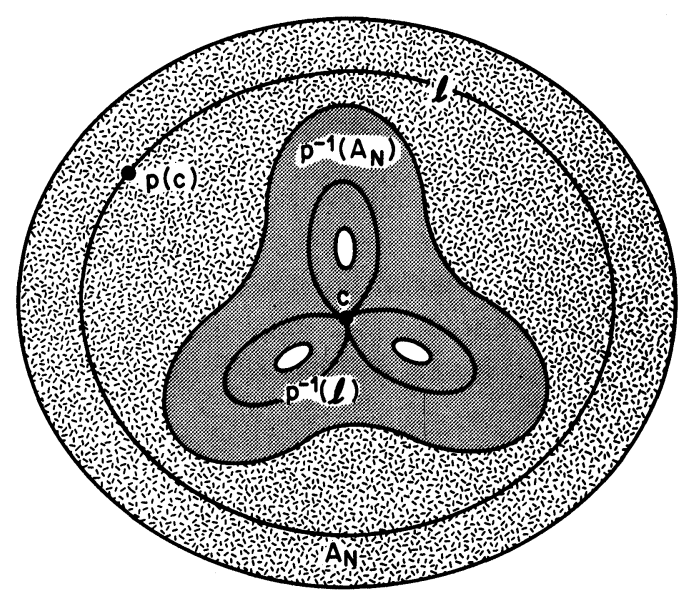

(9.7) Figure. The sets $p^{-1}\left(A_{N}\right)$ and $A_{N}$. 
We try to extend the conjugacy by taking inverse images by $p(z)$. Let $A_{-1}=p^{-1}\left(A_{0}\right)$. If $A_{0} \cap p\left(C^{\prime}\right)=\varnothing$, then $p: A_{-1} \rightarrow A_{0}$ is a $d$-fold covering space and $A_{-1}$ is an annulus. Hence, we can pull back the coordinate system and define a conjugacy $h: A_{-1} \rightarrow D_{s}$ where $s=r^{1 / d}$. We repeat this process inductively by defining $A_{-k}=p^{-1}\left(A_{-k+1}\right)$ as long as $A_{-k+1} \cap p\left(C^{\prime}\right)=\varnothing$.

Our assumption implies the existence of an integer $N$ such that $p(c) \in A_{N}$ for some $c \in C^{\prime}$. In this case the map $p: p^{-1}\left(A_{N}\right) \rightarrow A_{N}$ is not a covering space but is a branched cover. See Figure (9.7).

The inverse image of the Jordan curve $l$ through $p(c)$ is a pinched curve which bounds at least two finite, open sets, and these sets disconnect $J(p)$.

$4 \Rightarrow 1$. This implication is proved using exactly the same observations as in the proof of $3 \Rightarrow 4$. We are able to use the same notation for the annuli $A_{-k}$, and our assumption implies that $p: A_{-k} \rightarrow A_{-k+1}$ is always a $d$-fold covering space. Since $A(\infty)=U \cup\left(\cup_{n=1}^{\infty} A_{-n}\right)$, the conclusion follows immediately.

The other extreme-all the finite critical points have orbits asymptotic to infinity-has behavior which is quite different from the type described above. The Julia set is totally disconnected. In fact, we can give a complete topological description of the dynamics on the Julia set.

(9.8) DeFinition. Let

$$
\Sigma_{n}=\prod_{k=0}^{\infty}\{1, \ldots, n\} .
$$

The shift map $\sigma: \Sigma_{n} \rightarrow \Sigma_{n}$ is defined by

$$
\left[\sigma\left(\left\{s_{i}\right\}_{0}^{\infty}\right)\right]_{i}=s_{i+1} .
$$

The dynamical system $\sigma: \boldsymbol{\Sigma}_{n} \rightarrow \boldsymbol{\Sigma}_{n}$ is called a one-sided shift on $n$ symbols.

If one has not encountered this system before, one should note that it has rather complicated dynamics. It has lots of periodic orbits, and there are also dense orbits.

(9.9) ThEOREM. Suppose $C^{\prime} \subset A(\infty)$. Then $J$ is totally disconnected and $p \mid J$ is isomorphic to the one-sided shift on d symbols.

Figure (2.3) is a picture of the Julia set of a quadratic polynomial whose finite critical point (namely 0 in this case) has an orbit asymptotic to infinity.

The proof of this theorem is based on the same idea as the last proof-attempting to extend the conjugacy near infinity. Finite critical values cause pinching of the coordinate system. Actually there is so much pinching that $J$ must be totally disconnected.

Proof. We could simply give a proof that works in the general case, but to illustrate the above observations concerning extending conjugacies, we give a proof that works in the quadratic case before we do the general case.

Suppose $\operatorname{deg}(p)=2$. Just as in the last proof, we start with a conjugacy $h: U \rightarrow D_{r}$ between $p(z)$ and $z \mapsto z^{2}$ in a neighborhood of infinity, and we consider the annulus $A_{0}=h^{-1}\left(D_{r^{2}}-D_{r}\right)$. We can inductively define annuli 
$A_{-k}=p^{-1}\left(A_{-k+1}\right)$ as long as $A_{-k+1}$ does not contain the finite critical value of $p(z)$. If $p(c) \in A_{N}$ for $c \in C^{\prime}$, then $p^{-1}\left(A_{N}\right)$ is a disk minus two holes. See Figure (9.10).

Another way to view this is to regard the coordinate system as a height function. Then we have Figure (9.11).

The two holes in $p^{-1}\left(A_{N}\right)$ yield two inverse functions defined on $B=$ $\overline{\mathbf{C}}-\left[U \cup \cup_{k=N}^{-1} A_{k}\right]$. See Figure (9.12).

The inverse function $I_{i}: B \rightarrow D_{i}$ is an analytic homeomorphism. Consequently, $I_{i}\left(p^{-1}\left(A_{N}\right)\right)$ is also a disk minus two holes $(i=1$ and 2$)$. See Figure (9.13).

Given any element $\left\{s_{i}\right\} \in \Sigma_{2}$ we can define $\phi\left(\left\{s_{i}\right\}\right)$ to be the constant in the constant limit function of the normal family $\left\{I_{s_{1}}, I_{s_{2}} \circ I_{s_{1}}, I_{s_{3}} \circ I_{s_{2}} \circ I_{s_{1}}, \ldots\right\}$. This family limits to a constant function because the hypothesis to Lemma

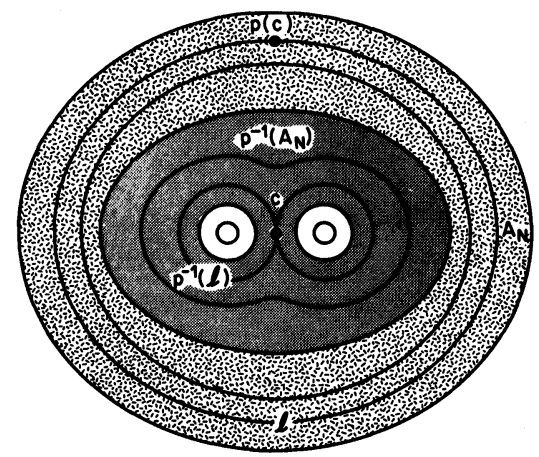

(9.10) FIGURE. The pinched foliation. Infinity is not in the picture.

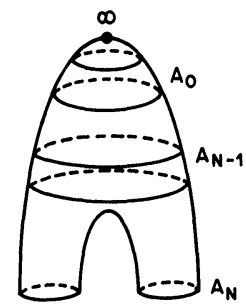

(9.11) Figure. A pair of pants with a cap.

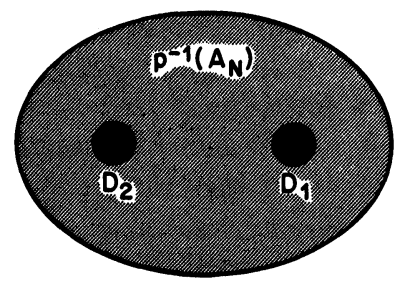

(9.12) FIGURE. The disk $B$ with the two disks $D_{1}$ and $D_{2}$ contained in it. 


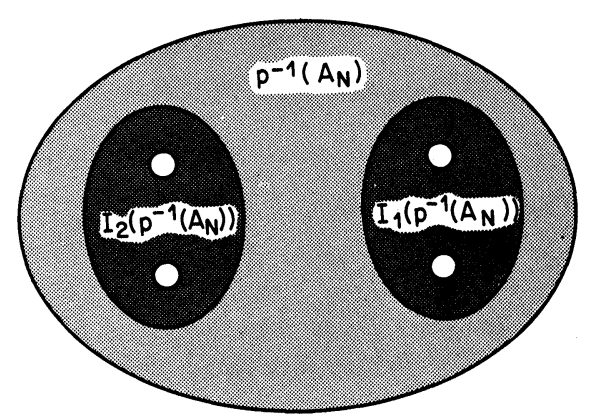

(9.13) FIGURE. Four eyes.

(8.2) is satisfied. We leave it to the reader to verify that $\phi$ is a topological conjugacy between $p \mid J$ and $\sigma \mid \Sigma_{2}$, i.e. $\phi$ is a homeomorphism and $\sigma \circ \phi=\phi \circ p$.

In the general case there can be more than one critical point in $C^{\prime}$. Connect $p\left(C^{\prime}\right)$ to infinity by disjoint paths-one for each element of $p\left(C^{\prime}\right)$-with the property that, if $B=\overline{\mathbf{C}}-\{$ paths $\}$, then $p^{-1}(B) \subset B$. The set $B$ is simply connected, and $d$ inverse functions $I_{i}: B \rightarrow B(i=1, \ldots, n)$ can be defined. A conjugacy $\phi: J \rightarrow \Sigma_{d}$ is defined just as above.

10. The Mandelbrot set and the work of Douady and Hubbard. Even a rational function as simple as a quadratic polynomial can be the source of complicated and intriguing dynamics, and there remain important unresolved questions regarding their dynamics. In this section we sketch the recent computer work of Mandelbrot and the results of Douady and Hubbard. These surprising results have been a major element in the explosion of interest in the subject during the last two years.

In Example (1.4) we showed how every quadratic polynomial is analytically conjugate to one of the form

$$
p_{c}(z)=z^{2}+c .
$$

Hence, the family of quadratics is really a one complex-dimensional family of dynamical systems. This normal form (10.1) is particularly handy because it permits accurate and complete computer studies relying on computer graphics. Another useful representation of the family of quadratics is

$$
f_{\lambda}(z)=\lambda z+z^{2} \text {. }
$$

Whereas the quadratic (10.1) always has the finite critical point located at the origin (so (10.1) is the best representation of the family to use if one is investigating questions concerning the forward orbits of critical points), the function (10.2) always has a fixed point at the origin whose eigenvalue is $\lambda$. Therefore, the form (10.2) is useful when studying bifurcation questions when $|\lambda|$ is near 1 . The reader should review the relationship between (10.1) and (10.2).

Theorems (9.5) and (9.9) yield a useful dichotomy between $c$ values for the family (10.1). If $0 \in A(\infty)$ or, equivalently, if the sequence $O^{+}(0)-0, c, c^{2}+$ $c,\left(c^{2}+c\right)^{2}+c, \ldots$-converges to infinity, then $J\left(p_{c}\right)$ is totally disconnected and $p_{c} \mid J$ is a one-sided shift on two symbols. On the other hand, if $0 \notin A(\infty)$, 
then $A(\infty)$ is simply connected and $J$ is connected. Mandelbrot [M2] investigated this dichotomy and found that it resulted in an unusual fractal.

(10.3) Definition. The Mandelbrot set $M$ is the subset of $\mathbf{C}$ defined as $\left\{c \in \mathbf{C} \mid J\left(p_{c}\right)\right.$ is connected $\}$.

The reader should note that this is the first set we have defined in a parameter space. Each point represents a different dynamical system. Figure (10.4) is the author's humble attempt to reproduce Mandelbrot's spectacular picture in [M1].

The reader may find it interesting to go back to the previous figures of Julia sets of quadratics and locate their $c$ values in (10.4). Many important open questions regarding quadratics are best phrased in terms of the Mandelbrot set, and some of these will be discussed in this section.

Computing pictures of the Mandelbrot set $M$ is tricky business. Initial computer studies indicated that $M$ had more than one "main body" and that it might be disconnected. In fact, it is connected.

(10.5) Theorem (Douady AND Hubbard [DH]). The Mandelbrot set is connected.

Douady and Hubbard prove (10.5) by constructing, using dynamics, a conformal automorphism $\psi: \overline{\mathbf{C}}-D_{1} \rightarrow \overline{\mathbf{C}}-M$. Hence, the complement of $M$ in the Riemann sphere is conformally equivalent to a disk in a dynamically natural way.

Douady and Hubbard have also analyzed the components of the interior of $M$. Let

$$
H=\left\{c \mid p_{c} \text { has a finite (super)attractive periodic orbit }\right\}
$$

Theorems (5.8) and (8.1) imply that, if $c \in H, p_{c} \mid J$ is expanding. It is routine to prove that $H \subset \operatorname{int}(M)$ and $H$ is open. The following conjecture has been the subject of much work in the last fifteen years.

(10.6) CONJECTURE. $H=\operatorname{int}(M)$.

Most workers believe (10.6) is true. However, until the problem is resolved, we must use some annoying terminology.

(10.7) Definition. A component $K$ of the interior of $M$ is hyperbolic if $K \subset H$.

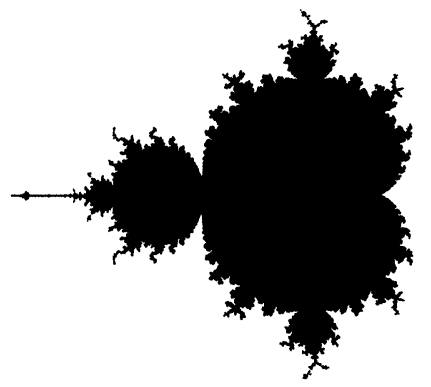

(10.4) Figure. The Mandelbrot set. 
Douady and Hubbard [DH] have also constructed a conformal representation of the hyperbolic components.

(10.8) THEOREM. Let $K$ be a hyperbolic component of the interior of $M$. Then $K$ is conformally equivalent to the disk $D_{1}$, and the equivalence may be given by the map $\rho: K \rightarrow D_{1}$ where $\rho_{K}(c)=\lambda_{c}$ and $\lambda_{c}$ is the eigenvalue of the unique finite (super) attracting periodic orbit of $p_{c}$.

The existence of such a simple conformal equivalence is quite surprising. It determines a center $\rho_{K}^{-1}(0)$ of each $K$, and we shall also talk about the root of $K$ which is the point $\lim _{t \in R \rightarrow 1} \rho_{K}^{-1}(t)$. (Douady and Hubbard prove that such a limit exists.)

Now we take a tour through $M$, and at each stop we describe the Julia set and its relationship with the Julia sets of other points in $M$. The tour starts at the origin, which is contained in a hyperbolic component that is actually the interior of a cardioid. Since $p_{0}(z)=z^{2}$, we are starting with the simplest Julia set in $M$. For $\varepsilon \neq 0$ but small, Sullivan [S4] proved that $J$ is still a Jordan curve, but its Hausdorff dimension must be greater than one. As we mentioned in (2.2), Ruelle [R] has shown that this dimension varies real analytically in the norm of the parameter if the parameter remains in a hyperbolic component of $M$. Figure (2.2) illustrates a typical Julia set in this component. Sullivan also established that $J$ is a quasi-circle.

We now move along the negative real axis until we stop at $c=-3 / 4$. We are at a boundary point between two hyperbolic components. This point is the root of the hyperbolic component containing $c=-1$. Figure (3.14) shows the Julia set of $p_{-3 / 4}$. This map does not have any (super)attracting periodic orbits. Oddly enough, it is the only quadratic polynomial of the form (10.1) with no periodic orbits of period two. The finite Sullivan domains are the two parabolic domains whose boundaries contain the neutral fixed point. They form a cycle of period two.

At $c=-1$ we are at the center of this hyperbolic component. Its Julia set is pictured in Figure (3.7). Since the centers of hyperbolic components are the $c$ values for which $p_{c}$ has a finite superattractive periodic orbit, the origin in $p_{-1}$ is part of a superattractive periodic orbit. The one-parameter family we have described so far (i.e. $t \rightarrow p_{t}$ with $t$ varying from 0 to -1 ) contains exactly one period doubling bifurcation. In other words, the period of the finite (super)attractive cycle has doubled.

If we continue along the negative real axis we encounter an infinite sequence of period doubling bifurcations - the Feigenbaum bifurcations. The reader should consult the book by Collet and Eckmann [CE] to learn more about these bifurcations.

Now we backtrack for a moment. Suppose, instead of leaving the origin along the negative real axis, we approached the boundary of the cardioid at the $c$ value

$$
c=\lambda / 2-\lambda^{2} / 4
$$


where $\lambda=e^{2 \pi i / 3}$, so that $p_{c}$ is conjugate to $f_{\lambda}$. Figure (3.15) is a picture of the Julia set of $f_{\lambda}$. See Figure (10.9).

Our journey so far has been entirely in the primary region of $M$. This primary region is inductively formed by starting with the cardioid $M_{0}$. At each successive step, an infinite number of hyperbolic components and their roots are added to $M_{i}$ to form $M_{i+1}$. A component $K$ is added to $M_{i}$ if its root is in the boundary of $M_{i}$. The primary region is the union $\bigcup_{i=0}^{\infty} M_{i}$. We do not get all of $M$ (by any means!) with this method. Suppose we had continued along the negative real axis. The corresponding systems would have undergone an infinite number of period doubling bifurcations. Yet, we would still be far from the end of $M \cap \mathbf{R}=[-2,1 / 4]$. Values of $c$ intervene where $p_{c} \mid J$ has "aperiodic" behavior. Then new secondary regions develop. Each of these has a main body which is something like a cardioid, and its structure is very similar to the primary body. Figures (10.10) and (10.11) locate one such secondary body and show it in more detail.

This computer evidence strongly suggests that the Mandelbrot set is also a fractal and that the dynamics always bifurcates according to the same "pattern". This is often referred to as "universality". Douady and Hubbard have results for polynomial-like mappings which support these ideas.

If $c \in M$ then $A(\infty)$ is simply connected and there exists a conjugacy $\phi_{c}: \overline{\mathbf{C}}-D_{1} \rightarrow A(\infty)$ such that $\phi_{c}(\infty)=\infty, \phi_{c}^{\prime}(\infty)=1$, and $\phi_{c}\left(z^{2}\right)=p_{c}\left(\phi_{c}(z)\right)$ for all $z \in \overline{\mathbf{C}}-D_{1}$ (see (9.5)). Much information can be gained by extending $\phi$ to the unit circle $S^{1}$. Extending Riemann maps is a classical aspect of complex analysis, and Carathéodory [Cd] obtained the relevant result.

(10.12) Definition. A subset $S \subset \overline{\mathbf{C}}$ is locally connected at $p \in S$ if, given any $\varepsilon>0$, there exists an $\varepsilon^{\prime}$ such that $0<\varepsilon^{\prime}<\varepsilon$ and $D_{\varepsilon^{\prime}}(p) \cap S$ is a subset of one component of $D_{\epsilon}(p) \cap S$.

(10.13) THEOREM (CARATHEODORY). Let $D$ be a simply connected domain which is conformally equivalent to the disk $D_{1}$. The boundary of $D$ is locally connected if and only if the conformal equivalence extends to a continuous map from $\overline{D_{1}}$ to $\bar{D}$.

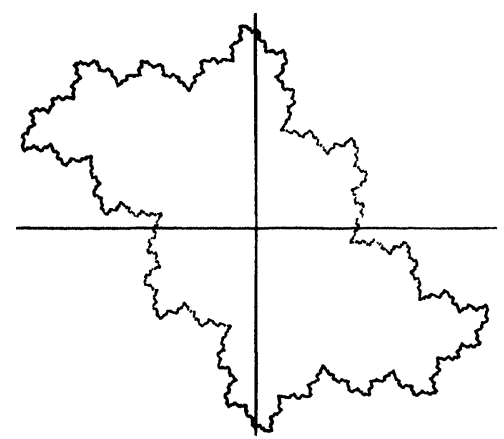

(10.9) Figure. The Julia set of $p_{\alpha}$ where $\alpha=.9 c$ and $c$ is defined by equation (10.8). This is the Julia set just before a period trebling bifurcation. We can see how the rabbit-like features of (2.1) evolve continuously from the Jordan curves of the main cardioid. 


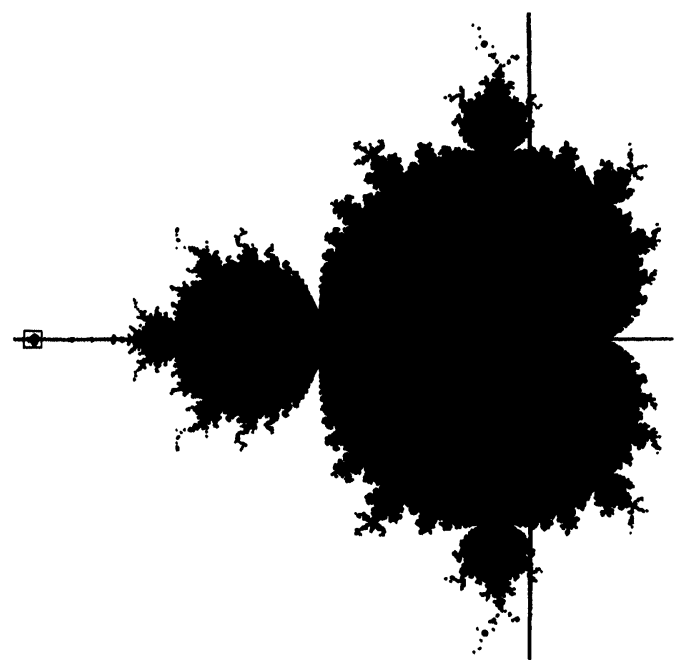

(10.10) Figure. A secondary body in the Mandelbrot set. This is $M$ with an additional small box centered on the negative real axis. This box encloses a secondary body. The box is magnified in Figure (10.11).

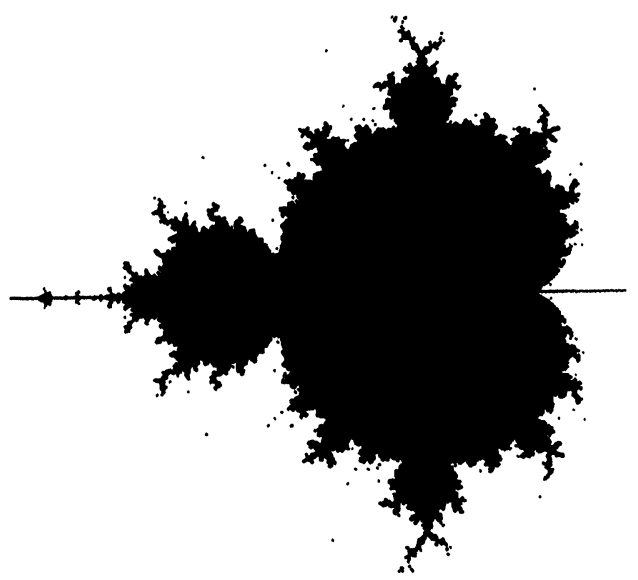

(10.11) FIGURE. An enlargement of the secondary body in (10.10).

Often the Julia set of a polynomial is locally connected and the map $\phi$ extends [D, DH, T1]. For example, if $c \in H$, then $J\left(p_{c}\right)$ is locally connected. Also, if $c$ is preperiodic, then $J\left(p_{c}\right)$ is a dendrite (see (7.11)). So there is a continuous map $\phi_{c}: S^{1} \rightarrow J\left(p_{c}\right)$ such that the diagram

$$
\begin{array}{ccc}
S^{1} & \stackrel{z \mapsto z^{2}}{\rightarrow} & S^{1} \\
\phi_{c} \downarrow & & \downarrow \phi_{c} \\
J\left(p_{c}\right) & \overrightarrow{p_{c}} & J\left(p_{c}\right)
\end{array}
$$


commutes. Since the map $\phi_{c}$ is not injective, it is not a conjugacy. Usually, it is called a semiconjugacy. Describing the map on the Julia set amounts to establishing the identifications made by the map $\phi_{c}$.

However, the Julia set is not always locally connected.

(10.14) THEOREM (DouAdy AND SUllivan [S4]). If $p_{c}$ has a neutral fixed point whose eigenvalue is not a root of unity and $p_{c}$ is not locally conjugate to its derivative, then $J\left(p_{c}\right)$ is not locally connected.

The proof requires a local analysis of "radial limits" to the fixed point. The arguments are similar to those Sullivan uses to show that orbits in parabolic domains are asymptotic to periodic points whose derivatives are roots of unity. The interested reader should consult [S4 and S2].

Since the exterior of $M$ in $\overline{\mathbf{C}}$ is also simply connected, the same questions apply to $\overline{\mathbf{C}}-M$. Douady and Hubbard [DH] have partial results regarding the Riemann map on the exterior of the Mandelbrot set.

(10.15) THEOREM. For all $\theta \in \mathbf{Q}$, the radial arc $r \rightarrow \psi\left(r e^{2 \pi i \theta}\right)$, with $r>1$, has a limit as $r \rightarrow 1$.

Given a map $p_{c}$ which is such a limit, Douady and Hubbard [DH] also construct an associated tree $H_{c}$ and a map on the tree. They give a combinatorial algorithm for determining $\theta$ from the map on $H_{c}$.

We conclude with one of the basic problems in the dynamical theory of quadratics.

(10.16) Problem. Is M locally connected?

11. The Measurable Riemann Mapping Theorem and analytic dynamics. In this section we sketch how the theory of quasi-conformal homeomorphisms has been applied to the study of one-dimensional, analytic dynamics. To do this we introduce (without proofs) the relevant ideas from the theory of quasi-conformal mappings and show how these notions can be used to create conformal maps with prescribed dynamics. These constructions are usually the key to any successful application of the Measurable Riemann Mapping Theorem.

We will need enough of the ideas from the theory of quasi-conformal homeomorphisms to be able to make sense of the statement:

(11.1) Any bounded measurable conformal structure on $\overline{\mathbf{C}}$ is quasi-conformally equivalent to the standard structure.

The fundamental underlying concept is the dilatation of a homeomorphism. If the map is also $C^{1}$, the dilatation is easy to define and its geometric interpretation is straightforward, so we do this case first.

Let $f: \mathbf{C} \rightarrow \mathbf{C}$ be an orientation preserving $C^{1}$ diffeomorphism. We can expand $f$ as

$$
f(z)=f\left(z_{0}\right)+f_{z}\left(z_{0}\right)\left(z-z_{0}\right)+f_{\bar{z}}\left(z_{0}\right)\left(\bar{z}-\bar{z}_{0}\right)+o\left(\left|z-z_{0}\right|\right),
$$


where

$$
f_{z}=\frac{1}{2}\left(f_{x}-i f_{y}\right) \text { and } f_{\bar{z}}=\frac{1}{2}\left(f_{x}+i f_{y}\right) .
$$

Note that, if $f(z)$ is analytic, then $f_{\bar{z}}=0$. With this notation the dilatation $D_{f}\left(z_{0}\right)$ of $f$ at $z_{0}$ is the quotient

$$
D_{f}\left(z_{0}\right)=\frac{\left|f_{z}\left(z_{0}\right)\right|+\left|f_{\bar{z}}\left(z_{0}\right)\right|}{\left|f_{z}\left(z_{0}\right)\right|-\left|f_{\bar{z}}\left(z_{0}\right)\right|}
$$

This quantity can be geometrically interpreted as follows. The derivative $d f\left(z_{0}\right)$ takes a circle in the tangent space of $\mathbf{C}$ at $z_{0}$ to an ellipse in the tangent space of $\mathbf{C}$ at $f\left(z_{0}\right)$. The dilatation is a measure of this distortion because it is the ratio of the length of the major axis to the length of the minor axis. The quantity

$$
K_{f}=\sup _{z \in \mathbf{C}} D_{f}(z)
$$

is called the maximal dilatation of $f$ on $\mathbf{C}$, and the map $f$ is called quasi-confor$m a l$ if $K_{f}<\infty$. Note that $f$ is conformal if and only if $K_{f}=1$.

In our context, it will be useful to use the quantity

$$
\mu_{f}=f_{\bar{z}} / f_{z} \text {. }
$$

One can verify that

$$
\left|\mu_{f}\right|=\left(D_{f}-1\right) /\left(D_{f}+1\right)<1 .
$$

If $f$ is quasi-conformal, then

$$
\left|\mu_{f}\right| \leqslant\left(K_{f}-1\right) /\left(K_{f}+1\right)<1 .
$$

We have introduced $\mu_{f}$ because the differential equation

$$
f_{\bar{z}}=\left(\mu_{f}\right) f_{z}
$$

is crucial to our discussion. It is called the Beltrami equation, and we need quasi-conformal homeomorphisms in order to solve it whenever $\mu$ is a measurable function with $\|\mu\|_{\infty}<1$.

The definition of the dilatation of a homeomorphism ((11.4)) is a great deal more complicated, and it is not immediately clear that it agrees with $D_{f}$ in the differentiable case. The two notions are related by Theorem (11.6).

(11.3) Definition. A quadrilateral $Q\left(z_{1}, z_{2}, z_{3}, z_{4}\right)$ is a Jordan domain in C with four distinguished boundary points: $z_{1}, z_{2}, z_{3}$, and $z_{4}$.

Using the theory of conformal mappings, one can assign a modulus $M(Q)$ to each quadrilateral $Q$. Choose a conformal map $\varphi$ of $Q$ to a rectangle $R$ such that $\varphi$ takes the distinguished points to the vertices of $R$. Then the modulus $M(Q)$ of $Q$ is the modulus $M(R)$, which is $a / b$. The modulus $M(Q)$ is well defined, and if $f: \mathbf{C} \rightarrow \mathbf{C}$ is conformal, $M(f(Q))=M(Q)$. See Figure (11.3a). 

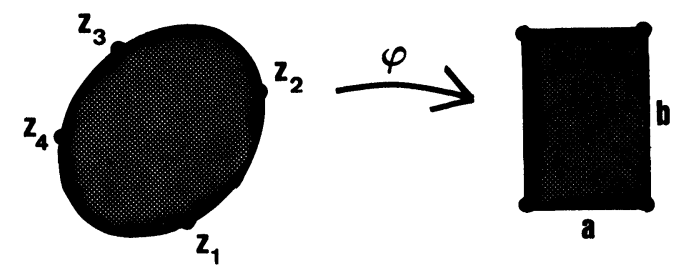

(11.3a) FIGURE

(11.4) Definition. Suppose $f: \mathbf{C} \rightarrow \mathbf{C}$ is an orientation-preserving homeomorphism. The quantity

$$
K_{f}=\sup _{Q \subset \mathbf{C}} \frac{M(f(Q))}{M(Q)},
$$

where the $Q$ 's range over all quadrilaterals, is the (maximal) dilatation of $f$. If $K_{f}<\infty, f$ is called a quasi-conformal homeomorphism.

(11.5) REMARK. Note that a conformal map has dilatation 1. It is also true that a quasi-conformal homeomorphism whose dilatation is 1 is conformal.

(11.6) THEOREM. A quasi-conformal homeomorphism $f$ with dilatation $K$ is differentiable almost everywhere, and $D_{f}\left(z_{0}\right) \leqslant K$ at each point $z_{0}$ where $f$ is differentiable.

Now we can state the Measurable Riemann Mapping Theorem. The reader should be aware of the fact that this theorem is commonly referred to as the Existence Theorem in the theory of quasi-conformal homeomorphisms. Our terminology is based on common usage in the study of complex dynamics rather than in the study of quasi-conformality.

(11.7) THEOREM. Let $\mu$ be a measurable function with $\|\mu\|_{\infty}<1$. Then there exists a quasi-conformal homeomorphism whose complex dilatation agrees with $\mu$ almost everywhere.

This theorem is the result of a great deal of work by a number of mathematicians. Since the author is not an expert in this subject, he does not feel capable of crediting everyone who should be mentioned. Moreover, the above discussion is far from complete, so the interested reader is referred to Ahlfors [A3] or Lehto and Virtanen [LV] for the details and bibliographic references.

Given these tools, we can make sense of (11.1). A conformal structure on $\mathbf{C}$ can be derived from a Riemannian metric

$$
d s^{2}=E d x^{2}+2 F d x d y+G d y^{2}
$$

because we can also write

$$
d s=\lambda(z)|d z+\mu(z) d \bar{z}|
$$

where $\lambda(z)>0$ and $\mu: \mathbf{C} \rightarrow \mathbf{C}$ with $|\mu(z)|<1$. The standard structure corresponds to the choices $E=G=1$ and $F=0$. Then $d s=|d z|$ and $\mu=0$. A 
bounded, measurable conformal structure corresponds to a choice of $\mu$ which satisfies the hypothesis of the Measurable Riemann Mapping Theorem ((11.7)). A map $w=f(z)$ will be a conformal map from the standard structure to the bounded, measurable structure almost everywhere if $f_{\bar{z}} / f_{z}=\mu$ almost everywhere. To see this we make the following calculation.

$$
|d w|=|d f|=\left|f_{z} d z+f_{\bar{z}} d \bar{z}\right|=\left|f_{z}\right|\left|d z+\left(f_{\bar{z}} / f_{z}\right) d \bar{z}\right| \text {. }
$$

Applying (11.7) to a bounded, measurable $\mu$ yields a quasi-conformal homeomorphism $f$ which is a conformal equivalence between the measurable conformal structure and the standard structure almost everywhere.

To illustrate how (11.1) is used to study the dynamics of rational maps, we discuss two applications. The first is Douady's and Hubbard's theory of polynomial-like functions (see [D]). We shall prove their basic result that the dynamics of a polynomial-like map is isomorphic to the dynamics of a polynomial, and we shall use this result to sharpen the bound on the number of nonrepelling periodic orbits of a polynomial. Secondly, we give a brief sketch of Sullivan's proof of the nonexistence of wandering domains ((7.1)).

Polynomial-like mappings have the same topological properties as polynomials, yet they also arise as restrictions of polynomials of higher degree or as restrictions of transcendental entire functions.

(11.8) Definition. Let $U^{\prime} \subset U$ be two simply connected domains in $\mathbf{C}$ such that $U^{\prime}$ is relatively compact in $U$. If $f: U^{\prime} \rightarrow U$ is a proper holomorphic map of degree $d$, then $f$ is a polynomial-like map of degree $d$ of $U^{\prime}$ into $U$.

Associated to any polynomial $p$ is a set $K_{p}$, called the filled-in Julia set of $p$, defined by

$$
K_{p}=\overline{\mathbf{C}}-W^{s}(\infty) \text {. }
$$

There is a corresponding notion for a polynomial-like map $f: U^{\prime} \rightarrow U$. Let

$$
K_{f}=\left\{z \in U^{\prime} \mid f^{n}(z) \in U^{\prime} \text { for } n=1,2,3, \ldots\right\} .
$$

The fundamental result in the theory of polynomial-like mappings is the fact that these maps have the dynamics of polynomials on their filled-in Julia sets.

(11.9) TheOREM (DouAdy AND HubBard [D]). Let $f: U^{\prime} \rightarrow U$ be a polynomial-like mapping of degree $d$. Then there exist a polynomial $p$ of degree $d$, neighborhoods $N_{1}$ of $K_{f}$ and $N_{2}$ of $K_{p}$, and a quasi-conformal homeomorphism $h$ : $N_{1} \rightarrow N_{2}$ such that $h(f(z))=p(h(z))$ for all $z \in N_{1}$ and such that $h_{\bar{z}}$ is identically zero on $K_{f}$.

Proof. Let $S$ be a subset of $U^{\prime}$ which is conformally equivalent to a disk and satisfies the following two conditions.

(11.10) The set $K_{f}$ is contained in the interior of $S$.

(11.11) The set $A=f(S)-S$ is diffeomorphic to an annulus $A^{\prime}$ of the form $D_{r^{d}}-D_{r}$ for some $r>1$. In fact, if $\alpha: \overline{\mathbf{C}}-D_{r} \rightarrow \overline{\mathbf{C}}-D_{r^{d}}$ denotes the map $z \mapsto z^{d}$, we want to choose the diffeomorphism $\varphi: A \rightarrow A^{\prime}$ so that $\varphi(f(z))=$ $\alpha(\varphi(z))$ for all $z \in \partial S$. 
Given (11.10) and (11.11) we define a bounded measurable structure on $\overline{\mathbf{C}}$ and a map g: $\overline{\mathbf{C}} \rightarrow \overline{\mathbf{C}}$ which preserves this structure. We view $\overline{\mathbf{C}}$ as the two-sphere formed from $f(S)$ and $\overline{\mathbf{C}}-D_{r}$ by identifying $z \in A$ with $\varphi(z)$ in $\overline{\mathbf{C}}-D_{r}$. With these identifications and (11.11), we can define a map $g: \overline{\mathbf{C}} \rightarrow \overline{\mathbf{C}}$ which equals $f$ on $S$ and $\alpha$ on $\overline{\mathbf{C}}-D_{r}$, and the bounded measurable structure is defined to be the standard structure on $\overline{\mathbf{C}}-D_{r}$ and $K_{f}$ and the pullback of the standard structure on $A$ by $f$ to $S-K_{f}$. It is important to note that, since $f$ is conformal, the pullback structure will be bounded. The map $g$ preserves this structure by definition.

When we apply (11.1) (which, as we have seen, is just (11.7) in different language), we get a quasi-conformal homeomorphism $h: \overline{\mathbf{C}} \rightarrow \overline{\mathbf{C}}$ and a map $p=h \circ g \circ h^{-1}$ which preserves the standard structure almost everywhere. Hence $p$ is conformal (see Ahlfors [A3]). The map $p$ is quasi-conformally conjugate to the map $z \mapsto z^{d}$ in a neighborhood of infinity, so $p$ is a polynomial of degree $d$. Moreover, $p$ restricted to a neighborhood of $K_{p}$ is quasi-conformally conjugate by $h$ to $f$ restricted to a neighborhood of $K_{f}$, and $h_{\bar{z}}=0$ on $K_{f}$.

REMARK. This proof contains the main ingredients of most applications of the Measurable Riemann Mapping Theorem to complex dynamics. One starts with a given conformal map, alters the standard structure in a bounded manner and, using (11.7), obtains a new conformal map quasi-conformally conjugate to the original with some desired property. In addition to Douady's paper [D], this type of argument is also found in $\$ 8$ of Sullivan [S1].

We have previously mentioned the following result (see \$9), and now we can see how it is an easy consequence of (11.9).

(11.12) CoRollaRY (DouAdy [D]). Let $p(z)$ be a polynomial of degree d. The number of finite nonrepelling periodic orbits is at most $d-1$.

Proof. Let $N$ be the set of nonrepelling periodic orbits of $p$. Construct a polynomial $q$ (of high degree) such that $q \mid N=0$, and $\left|(p+q)^{\prime}(z)\right|<1$ for all $z \in N$. Note that, for small $\varepsilon$, the map $f=p+\varepsilon q$ is polynomial-like of degree $d$ on some neighborhood $U$ of $K_{p}$. The points of $N$ will be attracting periodic orbits of $K_{f}$, and (5.8) combined with (11.9) implies that $f$ can have no more than $d-1$ attracting periodic orbits in $K_{f}$. Therefore, $N$ can only consist of $d-1$ nonrepelling orbits.

We end this section by sketching a special case of Sullivan's proof of (7.1). Suppose $W$ is a component of $F$ which is conformally equivalent to a disk and which is not eventually periodic. Since the number of critical points is finite, we can assume that the maps $R: W_{n} \rightarrow W_{n+1}$, where $W_{n}=R^{n}(W)$, are all injective. Sullivan produces a real analytic family of bounded measurable conformal structures on $U$, and the dimension of this family is larger than the dimension of the space of rational maps of degree $d$. He uses these structures to get a real analytic family of bounded, measurable conformal structures on $\overline{\mathbf{C}}$ by pushing forward and pulling back by $R$ on the forward and backwards orbits of $W$. The structure is extended to all of $\overline{\mathbf{C}}$ using the standard structure. As in the proof of (11.9), this Measurable Riemann Mapping Theorem yields a 
family of rational maps which are all quasi-conformally conjugate to $R$. Moreover, Sullivan constructs his family of bounded, measurable structures so that no two of the resulting rational maps are the same. Since the space of rational maps of degree $d$ now contains a subspace of higher dimension, the domain $W$ could not have existed in the first place.

12. Bibliographic notes. This paper is neither a complete exposition of the classical theory nor a comprehensive survey of the latest results. Instead, we have tried to present enough of both to give the reader a fair understanding of what is known, how it fits together, and some of the important questions which still need to be resolved. In the references we list many articles and books that have not been specifically cited in the text. They are included to give the reader a place to start in the library. But we must again emphasize that we have not made an exhaustive search of the literature. Omissions are the result of ignorance, not judgment, by the author. We end this paper by organizing some of these references.

There has been a tendency to look only at the latest or the original work without noting the work that took place from the thirties to the sixties. The reader who is interested in these results (in addition to Siegel's Theorem) can start by reading the papers of Cremer ([C] and others), Myrberg [My 1-3], Baker [Ba 1-11], and Töpfer [Tö]. Other references can be found in their bibliographies as well as the bibliography of Burckel [Bu].

We have not mentioned any of the ergodic theory of rational maps. Brolin's paper [B] contains some fundamental results, and recently the papers of Barnsley, Geronimo and Harrington [BGH 1-6] have studied the measures in question. The reader should definitely note the important papers of Rees [Re 1-2] in which she shows that the set of rational maps ergodic with respect to Lebesgue measure is a set with positive measure.

Rotation domains are a topic of vigorous research both by mathematicians and physicists. Herman [H1] has written a paper which involves the number-theoretic aspects of this topic. Relevant papers by physicists include Manton and Nauenberg [MN], Rüssman [Ru], Widom [W], and Widom, Bensimon, Kadinoff and Shenker [WBKS].

Newton's method is the source of another interesting collection of examples, and there is a surprising connection between Newton's method for cubics and the Mandelbrot set. This family is studied in depth by Curry, Garnett and Sullivan [CGS], and hopefully Hubbard will publish some of his amazing slides.

One can also study the dynamics of transcendental entire functions with exactly the same approach. However, since the phase space is not compact, some of the main results for rational maps do not hold in this case. Fatou [F4] discusses this somewhat, and Töpfer [Tö] wrote a relevant paper in the thirties. Lately, the subject has received much more attention. Baker has several papers on transcendental entire functions, and Misiurewicz [Mi], Devaney and Krych [DK], and Devaney [De] all study the family $\lambda e^{z}$.

Structural stability and rational maps is the topic of two important papers-Mañé, Sad, and Sullivan [MSS], and Sullivan and Thurston [ST]. A 
key step is the extension of quasi-conformal maps, and these papers have developed new tools which are also of interest in the theory of quasi-conformal homeomorphisms.

This subject was essentially ignored by most researchers in the area of dynamical systems for forty years. This changed when the papers of Guckenheimer [G] and Jakobson [Ja1, Ja2] were published. These three papers applied the modern theory of dynamical systems to the iteration of rational maps.

Finally, we mention the work of Thurston [T2] who has introduced the theory of Teichmüller space to this subject. He has a necessary and sufficient condition for the existence (in the space of rational maps) of a prescribed topological dynamical system. He also has quite a few new proofs of the classical results using the Poincare metric. The exposition [T2] will have significant impact.

\section{List of Notation}

All notation is listed under the section in which it is introduced.

$\overline{\mathbf{C}}$
$R: \overline{\mathbf{C}} \rightarrow \overline{\mathbf{C}}$
$S^{2}$
$\operatorname{deg}(R)$
$z_{n+1}=R\left(z_{n}\right)$
$O^{+}\left(z_{0}\right)$
$R^{n}$
$D_{r}$ or $D_{r}(z)$

$\S 1$

the Riemann sphere

a rational map

the two-sphere

the degree of $R$

the iterate of $z_{n}$

the forward orbit of $z_{0}$

the $n$th iterate of $R$ (under composition)

the open disk centered at the origin (or at $z$ )

of radius $r$ in the Euclidean metric

$\S 2$

$F$ or $F(R)$

$J$ or $J(R)$

$\S 3$

$\lambda$ or $\lambda_{z_{0}}$

(SFE)

$\Lambda$

the Fatou set (of $R$ ), otherwise known as the domain of equicontinuity

the Julia set (of $R$ )

the eigenvalue (of the periodic orbit $z_{0}$ )

the $k$ th derivative of $f$

the Schröder Functional Equation

the complex numbers of absolute value one

which satisfy the hypothesis of Siegel's

theorem

$E$ or $E_{z}$

the exceptional points (of the point $z$ ) 


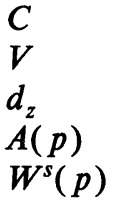

$O^{+}(C)$

$C^{\prime}$

$\Sigma_{n}$

$\boldsymbol{\sigma}$

$M$

H

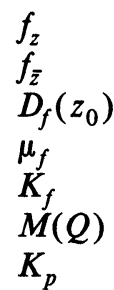

$\S 5$

the set of critical points

the set of critical values

the deficiency of the value $z$

the immediate attractive basin of $p$

the stable set of $p$

$\$ 8$

the forward orbits of the set of critical points

$\S 9$

the set of finite critical points of a polynomial

the space of one-sided sequences of $n$ symbols the shift map on $\Sigma_{n}$

\section{$\S 10$}

the Mandelbrot set

the union of hyperbolic components of $M$

$\S 11$

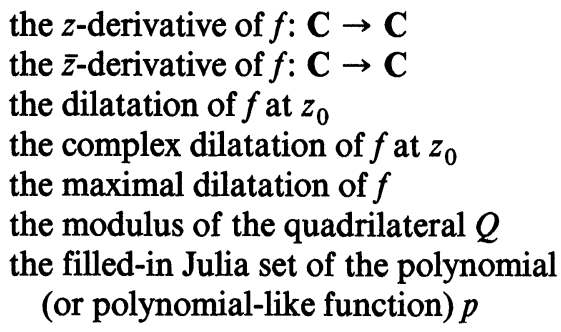

REFERENCES

[A1] L. Ahlfors, Complex analysis, McGraw-Hill, 1979.

[A2] __, Conformal invariants: Topics in geometric function theory, McGraw-Hill, 1973.

[A3] __ Lectures on quasiconformal mappings, Van Nostrand, 1966.

[Ab1] W. Abikoff, The real analytic theory of Teichmüller space, Lecture Notes in Math., vol. 820, Springer-Verlag, 1980.

[Ab2] _ The uniformization theorem, Amer. Math. Monthly 88 (1981), 574-592.

[Ar] V. Arnold, Small denominators. I: On the mappings of the circumference onto itself, Amer. Math. Soc. Transl. (2) 46 (1965), 213-284.

[B] H. Brolin, Invariant sets under iteration of rational functions, Ark. Mat., 6 (1965), 103-144.

[Ba1] I. Baker, The existence of fixpoints of entire functions, Math. Z. 73 (1960), 280-284.

[Ba2] __ Multiply connected domains of normality in iteration theory, Math. Z. 81 (1963), 206-214.

[Ba3] , Fixpoints of polynomials and rational functions, J. London Math. Soc. 39 (1964), 
[Ba4] 499-502. Sets of non-normality in iteration theory, J. London Math. Soc. 40 (1965),

[Ba5] _ The distribution of fixpoints of entire functions, Proc. London Math. Soc. (3) 16 (1966), 493-506.

[Ba6] _ Repulsive fixpoints of entire functions, Math. Z. 104 (1968), 252-256.

[Ba7] _ Limit functions and sets of non-normality in iteration theory, Ann. Acad. Sci. Fenn. Ser. A 467 (1970), 1-11.

[Ba8] __ Completely invariant domains of entire functions, Mathematical Essays Dedicated to A. J. MacIntyre, Ohio Univ. Press, 1970.

[Ba9] (1975), 277-283.

[Ba10] _, An entire function which has wandering domains, J. Austral. Math. Soc. (A) 22 (1976), 173-176.

[Ba11] _ The iteration of polynomials and transcendental entire functions, J. Austral. Math. Soc. (A) 30 (1981), 483-495.

[BGH1] M. Barnsley, J. Geronimo and A. Harrington, Some treelike Julia sets and Pade approximants (preprint).

[BGH2] _ Infinite dimensional Jacobi matrices associated with Julia sets, Proc. Amer. Math. Soc. (to appear).

[BGH3] , Geometry, electrostatic measure and orthogonal polynomials on Julia sets for polynomials (preprint).

[BGH4] _ Orthogonal polynomials associated with invariant measures on Julia sets, Bull. Amer. Math. Soc. (N.S.) 7 (1982), 381-384.

[BGH5] _ On the invariant sets of a family of quadratic maps, Comm. Math. Phys. (to appear).

[BGH6]__ Geometrical and electrical properties of some Julia sets (preprint).

[BH] M. Barnsley and A. Harrington, Moments of balanced measures on Julia sets (preprint).

[BL] I. Baker and L. Liverpool, The value distribution of entire functions of order at most one, Acta Sci. Math. 41 (1979), 3-14.

[Bo] Boettcher, Bull. Kasan Math. Soc. 14 (1905), 176. 396-407.

[Bu] R. Burckel, Iterating analytic self-maps of discs, Amer. Math. Monthly 88 (1981),

[C] H. Cremer, Zum Zentrumproblem, Math. Ann. 98 (1928), 151-163.

[Ca] C. Camacho, On the local structure of conformal mappings and holomorphic vector fields, Astérisque 59-60 (1978), 83-94.

[Cd] C. Caratheodory, Theory of functions, Vols. 1, 2, Chelsea, New York, 1960. 1980.

[CE] P. Collet and J. Eckmann, Iterated maps of the interval as dynamical systems, Birkhäuser,

[CH] S. Chow and J. Hale, Methods of bifurcation theory, Springer-Verlag, 1982.

[CGS] J. Curry, L. Garnett and D. Sullivan, On the iteration of a rational function computer experiments with Newton's method, Comm. Math. Phys. 91 (1983), 267-277.

[Co] H. Cohn, Conformal mapping on Riemann surfaces, McGraw-Hill, 1967.

[Co1] P. Collet, Local $C^{\infty}$ conjugacy on the Julia set for some holomorphic perturbations of $z \rightarrow z^{2}$, Preprint No. 18, Inst. Math. and Appl. Minneapolis, Minn., 1983.

[D] A. Douady, Systems dynamiques holomorphes, Séminaire Bourbaki, $35^{\mathrm{e}}$ année, 1982/83, No. 599, 1982.

[De] R. Devaney, The structural instability of $\operatorname{Exp}(z)$ (preprint).

[DH] A. Douady and J. Hubbard, Itération des polynômes quadratiques complexes, C. R. Acad. Sci. Paris 294 (1982), 123-126.

[DK] R. Devaney and M. Krych, Dynamics of $\exp (z)$, Ergodic Theory Dynamical Systems (to appear).

[dL] R. de la Llave, A simple proof of C. Siegel's center theorem, Preprint No. 2, Inst. Math. and Appl., Minneapolis, Minn., 1983.

[F1] P. Fatou, Sur les equations fonctionnelles, Bull. Soc. Math. France 47 (1919), 161-271.

[F2] __ Sur les equations fonctionnelles, Bull. Soc. Math. France 48 (1920), 33-94.

[F3] __ Sur les equations fonctionnelles, Bull. Soc. Math. France 48 (1920), 208-314. 
[F4] , Sur l'itération des fonctions transcendantes entières, Acta Math. 47 (1926), $337-370$ 1980.

[FK] H. Farkas and I. Kra, Riemann surfaces, Graduate Texts in Math., vol. 71, Springer-Verlag,

[G] J. Guckenheimer, Endomorphisms of the Riemann sphere (S. S. Chern and S. Smale, eds.), Proc. Sympos. Pure Math., vol. 14, Amer. Math. Soc., Providence, R. I., 1970.

[H1] M. Herman, Exemples de fractions rationelles ayant une orbite dense sur la sphere Riemann (preprint). [H1].

[H2] __ Lecture notes on an easy proof of Siegel's theorem. See also Appendice VII of

[Hi] E. Hille, Analytic function theory, Vols. 1, 2, Chelsea, New York, 1973.

[J] G. Julia, Memoire sur l'iteration des fonctions rationnelles, J. Math. 8 (1918), 47-245. See also Oeuvres de Gaston Julia, Gauthier-Villars, Vol. I, Paris, 121-319.

[Ja1] M. Jakobson, Structure of polynomial mappings on a singular set, Mat. Sb. 80 (1968), 105-124; English transl. in Math. USSR-Sb. 6 (1968).

[Ja2] _ On the problem of the classification of polynomial endomorphisms of the plane, Mat. Sb. 80 (1969), 365-387; English transl. in Math. USSR-Sb. 9 (1969).

[L] S. Lattès, Sur l'itérartion des substitutions rationnelles et les fonctions de Poincaré, C. R. Acad. Sci. Paris 166 (1918), 26-28.

[LV] O. Lehto and K. Virtanen, Quasiconformal mappings in the plane, Springer-Verlag, 1973.

[M1] B. Mandelbrot, The fractal geometry of nature, Freeman, 1982.

[M2] _ Fractal aspects of the iteration of $z \rightarrow \lambda z(1-z)$ and $z$, Ann. New York Acad. Sci. 357 (1980), 249-259.

[MN] N. S. Manton and M. Nauenberg, Universal scaling behavior for iterated maps in the complex plane (preprint).

[Mi] M. Misiurewicz, On iterates of $e^{z}$, Ergodic Theory Dynamical Systems 1 (1981), 103-106.

[Mo] P. Montel, Lecons sur les familles normales, Gauthier-Villars, Paris, 1927.

[Moe] R. Moeckel, Rotations of the closures of some simply connected domains, Univ. of Minnesota Math. Report 83-121.

[MSS] R. Mañé, P. Sad and D. Sullivan, On the dynamics of rational maps (preprint).

[My1] P. Myrberg, Inversion der Iteration fur rationale Funktionen, Ann. Acad. Sci. Fenn. Ser. A 292 (1960).

[My2] __, Sur l'iteration des polynomes reels quadratiques, J. Math. Pures Appl. (9) 41 (1962), 339-351. (1964).

[My3] __ Iteration der polynome mit reellen Koeffizienten, Ann. Acad. Sci. Fenn. Ser A 348

[N] M. Narasimhan, Riemann surfaces, Tata Inst. Fund. Res., Math. Pamphlets No. 1, 1963.

[Ne] M. Newman, Elements of the topology of plane sets, Cambridge Univ. Press, 1939.

[Ni] I. Niven, Irrational numbers, Math. Assoc. Amer., Washington, D. C., 1956. 99-108.

[R] D. Ruelle, Repellers for real analytic maps, Ergodic Theory Dynamical Systems 2 (1982),

[Re1] M. Rees, Ergodic rational maps with dense critical point forward orbit, Univ. of Minnesota Math. Report 82-140.

[Re2] _ Positive measure sets of ergodic rational maps, Univ. of Minnesota Math. Report 82-169.

[Ri] J. Ritt, On the iteration of rational functions, Trans. Amer. Math. Soc. 23 (1920), 348-356.

[Ro] P. Rosenbloom, L'iteration des fonctions entieres, C. R. Acad. Sci. Paris 9 (1948), 382-383.

[Ru] H. Rüssman, Kleine Nenner. II: Bemerkungen zur Newtonschen Method, Nachr. Akad. Wiss. Göttingen Math. Phys. K1 (1972), 1-20.

[S1] D. Sullivan, Quasiconformal homeomorphisms and dynamics. I (preprint).

[S2] _ Quasiconformal homeomorphisms and dynamics. III: Topological conjugacy classes of analytic endomorphisms (preprint). 1982.

[S3] _ Seminar on conformal and hyperbolic geometry, IHES Seminar notes, March , Conformal dynamical systems (preprint). 
[Si] C. Siegel, Iteration of analytic functions, Ann. of Math. (2) 43 (1942), 607-612.

[SM] C. Siegel and J. Moser, Lectures on celestial mechanics, Springer-Verlag, 1971.

[ST] D. Sullivan and W. Thurston, Extending holomorphic motions (preprint).

[T1] W. Thurston, Lecture notes from seminar at Princeton University.

[T2] __ Lecture notes, CBMS Conf. Univ. of Minnesota, Duluth, Minn.

[Tö] H. Töpfer, Über die Iteration der gazen transzendenten Funktionen, insbesondere von $\sin z$ und $\cos z$, Math. Ann. 117 (1939), 65-84.

[W] M. Widom, Renormalization group analysis of quasi-periodicity in analytic maps, Comm. Math. Phys. 92 (1983), 121-136.

[WBKS] M. Widom, D. Bensimon, L. Kadinoff and S. Shenker, Strange objects in the complex plane (preprint).

[Z] E. Zehnder, A simple proof of a generalization of a theorem of C. L. Siegel, Lecture Notes in Math., vol. 597, Springer-Verlag, 1977, pp. 855-866.

Department of Mathematics, Boston University, Boston, MassachusetTs 02215 
